\title{
A Global Temporal Genetic Program for Neural Circuit Formation
}

Saumya Jain ${ }^{1,4}$, Ying Lin ${ }^{1,2,4}$, Yerbol Kurmangaliyev ${ }^{3}$, Parmis Mirshahidi ${ }^{1}$, Brianna Parrington ${ }^{1}$, and S. Lawrence Zipursky ${ }^{1,3, *}$

${ }^{1}$ Department of Biological Chemistry, University of California, Los Angeles, USA. ${ }^{2}$ Molecular Biology Institute, University of California, Los Angeles, USA. ${ }^{3}$ Howard Hughes Medical Institute, David Geffen School of Medicine, University of California, Los Angeles, USA. ${ }^{4}$ These authors contributed equally to this work. "e-mail: 1zipursky@mednet.ucla.edu 
Wiring a complex brain relies on cell-type and temporal-specific expression of genes encoding cell recognition molecules regulating circuit formation ${ }^{1-3}$. Though genetic

3 programs driving cell-type specificity have been described $^{4-6}$, how precise temporal control

4 is achieved remains unknown. Here, we describe a global program for the temporal

5 regulation of cell-type-specific wiring genes in the Drosophila visual system. We show that

6 the Ecdysone Receptor induces a synchronous cascade of transcription factors in neurons

7 throughout the visual system in a highly stereotyped fashion. Single-cell RNA-seq analysis of

8 neurons lacking transcription factors in the cascade revealed that targets are cell-type

9 dependent and these are enriched for wiring genes. Knock-down of different transcription

10 factors in this cascade led to defects in sequential steps in wiring. Taken together, this work

11 identifies a synchronous, global program for temporal control of different sets of wiring

12 genes in different neurons. We speculate that this global program coordinates development

13 across cell types to choreograph specific steps in circuit assembly. 


\section{MAIN}

Animal behavior is dependent upon the formation of neuronal circuits with high fidelity.

16 Many cell-surface proteins, particularly of the Immunoglobulin (Ig), Leucine-Rich Repeat and

17 Cadherin families, promote interactions between neurons during circuit assembly ${ }^{1,7}$. Several

18 developmental strategies have evolved to meet the demands of cellular recognition specificity

19 during circuit construction. These include the expression of combinations of different recognition

20 molecules in different neurons, and reusing the same molecules to determine multiple, spatially

21 and temporally separated neuron-neuron interactions $s^{2,3,8}$. Wiring specificity, thus, relies critically

22 on genetic programs which regulate the cell-type and temporal specificity of genes encoding

23 recognition molecules. Many programs that define cell-type specificity have been described ${ }^{4-6}$.

24 Here we set out to assess how temporal specificity is determined.

\section{Dynamic expression of wiring genes}

To identify genetic programs regulating the temporal control of wiring genes, we profiled

27 the transcriptomes of post-mitotic lamina neurons (L1-L5) in the Drosophila optic lobe (analogous

28 to bipolar cells in the mammalian retina ${ }^{8}$ ), every $12 \mathrm{~h}$ from $24 \mathrm{hAPF}$ (hours After Pupa Formation)

29 to adults (Fig. 1a, b). These time points encompass all aspects of circuit formation for lamina

30 neurons once their growth cones have reached their target neuropil ${ }^{9,10}$. For transcriptional

31 profiling, we modified a recently developed single-cell RNA-Seq-based approach that utilizes the

32 natural genetic variation (i.e detected by single nucleotide polymorphisms (SNPs)) amongst

33 different Drosophila wildtype lines $\left(\mathrm{DGRP}^{11}\right)$ to determine gene expression in many different

34 samples and different time points in one experiment (Kurmangaliyev et al. revision under review,

35 Fig. 1b, Extended data Fig. 1, Supplementary Table 1). This approach eliminates issues due to 
36 batch effects that limit transcriptome analyses combining data from independent experiments.

37 Using this approach, we acquired transcriptomic data for 8269 cells distributed between seven

38 timepoints (24hAPF - Adult) (Fig. 1b, Extended data Fig. 1b, Supplementary Table 2). Using the

39 SNPs within the transcripts encoded by the different DGRP strains, we assigned each cell to a

40 specific time point. We integrated all cells and performed unsupervised clustering independent of

41 global changes occurring over time yielding five clusters (see Methods). We used previously

42 identified lamina neuron-type specific genes to assign each cluster to a cell-type ${ }^{12}$ (Extended data

43 Fig. 1c). For all subsequent analyses, we used normalized gene expression values prior to

44 integration. The expression patterns were similar to expression data from bulk RNA-seq (this

45 study, Supplementary Table 3) and from a large single cell sequencing study of visual system

46 neurons from our laboratory (Kurmangaliyev et al. revision under review) (Extended data Fig. 2).

47 Application of k-means clustering to group genes based on their expression dynamics

48 revealed several groups that showed dynamic patterns of expression (e.g. gene groups 5, 7, 9 and

4910 in Fig. 1c; also see Extended data Fig. 3 and Supplementary Table 4). Dynamically expressed

50 genes (especially early-peaking gene groups) were enriched (over expected by chance) for genes

51 encoding Immunoglobulin Superfamily (IgSF) proteins, as well as GO terms related to wiring (Fig.

52 1c, Extended data Fig. 3, Supplementary Table 5). Genes encoding IgSF proteins expressed in a

53 dynamic temporal fashion (see Methods), were also expressed in a cell-type-dependent manner

54 (Extended data Fig. 4a). Thus, as previously described ${ }^{13,14}$, wiring genes are expressed in a cell-

55 type and temporally dynamic fashion during circuit assembly. 


\section{Synchronous expression of the EcR Cascade}

To identify transcription factors (TFs) that drive gene expression in a dynamic fashion, we

58 looked at TFs that were differentially expressed between pairs of consecutive time points for each

59 neuron-type across development (Extended data Fig. 4b, see Methods). Interestingly, for each

60 neuron-type, dynamically expressed TFs were enriched for the Nuclear Receptor Transcription

61 Factor Pathway (Reactome Pathway Enrichment Analysis ${ }^{15}$, p-value $<10^{-5}$, Extended data Fig.

62 4c). These TFs, including the Ecdysone Receptor (EcR) and others regulated by Ecdysone ${ }^{16,17}$,

63 exhibit synchronous patterns of expression in lamina neurons over development (red lines in Fig.

64 2a, Extended data Fig. 5a, b). In addition, EcR also undergoes a temporal-specific isoform switch

65 due to the use of an alternative transcription start site, from predominantly EcR-B1 to EcR-A in

66 these neurons (Fig. 2a, Extended data Fig. 5), as determined by staining with isoform-specific

67 antibodies and bulk RNA sequencing. This temporal switch in isoform expression has been

68 reported previously for other neurons in the central nervous system ${ }^{18}$.

70 analysis of the whole optic lobe transcriptome (Kurmangaliyev et al. revision under review)

71 revealed that this transcriptional cascade has near-synchronous dynamics in all optic lobe neurons

72 throughout development (Fig. 2a). The timing and order of expression of these TFs correlates with

73 a mid-pupal pulse of Ecdysone ${ }^{16,17,19-21}$ (Fig. 2a). A role for this transcriptional cascade in various

74 developmental contexts, including neuronal remodeling of specific neuron-types during

75 metamorphosis has been studied in detail ${ }^{22,23}$. The coordinate and visual-system-wide expression

76 of these transcription factors during pupal development raised the intriguing possibility that this

77 cascade acts as a temporal regulator of circuit formation more broadly. 


\section{Temporal control of cell-type specific wiring genes}

We next sought to address whether the EcR cascade drives the temporal expression of genes in lamina neurons. Analysis of putative cis-regulatory regions ${ }^{24}$ for dynamically expressed

81 genes in lamina neurons showed a significant enrichment for binding sites for TFs in the EcR

82 cascade (Supplementary Table 6, see Methods), consistent with their direct role in driving these

83 patterns of gene expression. Additionally, ATAC-Seq ${ }^{25}$ at $40 \mathrm{hAPF}, 60 \mathrm{hAPF}$ and $72 \mathrm{hAPF}$ in L1

84 neurons (see Methods and Extended data Fig. 6, Supplementary Table 7) identified an enrichment

85 for predicted binding sites for TFs in this cascade (e.g. EcR, Hr3, Hr4 and Ftz-f1) in regions with

86 dynamic patterns of accessibility ${ }^{24}$ (Extended data Fig. 6d, Supplementary Table 6). This is

87 consistent with these TFs controlling chromatin structure, transcription of specific genes or both ${ }^{26}$.

88 To identify genes regulated by the EcR cascade, we targeted expression of a dominant

89 negative allele of EcR $\left(\mathrm{EcR}^{\mathrm{DN}}\right.$, EcR-B1 W650A $\left.{ }^{27}\right)$, EcR RNAi and Hr3 RNAi to lamina neurons.

90 Transcriptional profiling was performed using a single-cell RNA-Seq-based assay (Fig. 2b) to

91 compare transcriptomes of wildtype and mutant lamina neurons at four timepoints (24hAPF,

92 48hAPF, 72hAPF and Adult) in a pooled fashion using the DGRP strategy (Supplementary Table

93 1). By comparing the transcriptomes of 3606 wildtype and $1503 \mathrm{EcR}^{\mathrm{DN}}$-expressing lamina

94 neurons, we identified 921 differentially expressed genes (fold change $\geq 2$, p-value $\leq 0.05$ ), out of

95 a total of 3200 genes expressed in lamina neurons across development. These were distributed

96 between the five lamina neuron-types and four time-points (Extended data Fig. 7, Supplementary

97 Table. 8). Importantly, $\mathrm{EcR}^{\mathrm{DN}}$-expressing lamina neurons continued to express their cell-type

98 specific TFs, suggesting no changes in cell-fate (Extended date Fig. 7b). In contrast to L1 and L3-

$99 \mathrm{~L} 5, \mathrm{EcR}^{\mathrm{DN}}$ had modest effects on gene expression in L2. This is consistent with lower EcR ${ }^{\mathrm{DN}}$

100 expression in L2 neurons at 48hAPF using this GAL4 driver line (Extended data Fig. 7e). Analysis 
101 of the 921 differentially expressed transcripts led to four conclusions. First, EcR-dependent genes

102 are enriched for those showing dynamic expression in wildtype cells (Extended data Fig. 7f, 11).

103 Second, a large set of genes dependent upon this cascade is expressed in a cell-type-dependent

104 fashion in wildtype lamina neurons (Fig. 2c, Extended data Fig. 8). Third. the cell-type dependent

105 genomic targets of this cascade were highly enriched for IgSF genes (Hypergeometric test, p-value

$106=1.25 \times 10^{-18}$, see Methods) as well as GO terms associated with wiring (Fig. 2d, e, Supplementary

107 Table 9). And finally, it was not uncommon for $\mathrm{EcR}^{\mathrm{DN}}$ to have different effects on the same gene

108 in different neuron types. This may reflect different levels of expression of $\mathrm{EcR}^{\mathrm{DN}}$. In some cases,

109 however, opposite effects in different lamina neurons were observed (Fig. 2d).

110 Three lines of evidence indicated that genes affected by the expression of EcR ${ }^{\mathrm{DN}}$ were

111 largely bona fide targets of this transcriptional cascade. First, most genes affected by EcR ${ }^{\mathrm{DN}}$ were

112 affected in a similar way by EcR RNAi, although the change was typically less pronounced

113 (Extended data Fig. 9, 12a; Supplementary Table 10). Second, changes in gene expression in Hr3

$114 \mathrm{RNAi}$ and $\mathrm{EcR}^{\mathrm{DN}}$ were similar, especially at later time points, as expected from their genetic

115 relationship (Fig. 2a; Extended data Fig. 10, 12b; Supplementary Table 11). And third, expression

116 of several putative EcR-dependent genes in brains cultured ex vivo ${ }^{28}$ depended on the inclusion of

117 Ecdysone in the medium (see Methods, Extended data Fig. 13, 16b). Thus, EcR, either directly or

118 indirectly, controls the temporal expression of wiring genes in a cell-type-specific manner.

119 The cell-type-specific, temporal control of wiring genes by the EcR cascade suggests that

120 it plays a role in circuit formation. Consistent with this notion, we observed a disorganization of

121 lamina neuron arbors in the medulla neuropil upon pan-lamina expression of EcR ${ }^{\mathrm{DN}}$, which was

122 largely rescued by overexpression of wildtype EcR-B1 (Extended data Fig. 14a). A disorganized

123 neuropil was also seen upon targeted expression of EcR RNAi. Disruption of the axon terminal 
morphology of T4/T5 neurons in the lobula plate neuropil, a more central visual system structure, was also seen upon targeted expression of $\mathrm{EcR}^{\mathrm{DN}}$ to these neurons (Extended data Fig. 14b). Together these data support the idea that the EcR transcriptional cascade regulates genetic

127 programs governing the appropriate expression of cell surface and secreted proteins required for 128 proper wiring of the visual system.

\section{EcR regulates the L3-specific expression of Netrin}

We next sought to assess whether loss of EcR function gave rise to similar phenotypes seen

131 for loss-of-function mutations in cell surface or secreted proteins regulating wiring in L1-L5. Loss

132 of EcR activity resulted in phenotypes similar to Netrin mutants in L $3^{29,30}$ (Fig. 3a-d) and mutations

133 in $D I P$ - $\beta$, encoding an IgSF protein, in $\mathrm{L}^{31}$ (Extended data Fig. 15). Here we focus on the

134 relationship between $E c R$ and Netrin.

135 Netrin protein is required in L3 axon terminals in the M3 layer to promote adhesion of

136 photoreceptor R8 growth cones to processes in the same layer. In Netrin mutants, R8 terminals

137 extend into the developing M3 layer, but are unable to stably adhere to it and retract to the surface

138 of the medulla ${ }^{30}$. At least one other, yet to be identified, signal is necessary for R8 to recognize the

$139 \mathrm{M} 3$ layer $^{30}$. From sequencing of mutants and anti-Netrin antibody staining, EcR activity is required

140 for Netrin expression in L3 (Fig. 3a, b, Extended data Fig. 16b). Targeting expression of EcR $\mathrm{R}^{\mathrm{DN}}$ in

141 L3 leads to an R8 mis-targeting phenotype. Here most R8 terminals extend beyond the M3 layer

142 and some remain at the surface of the medulla. The EcR ${ }^{\mathrm{DN}}$ phenotype is partially rescued by

143 overexpression of Netrin in developing EcR-mutant L3 neurons (Fig. 3c). Differences between the

144 Netrin mutant and $\mathrm{EcR}^{\mathrm{DN}}$ phenotypes are consistent with the notion that EcR regulates one or more

145 signals in L3, in addition to Netrin, necessary for R8 targeting ${ }^{30}$ (Fig. 3d). 
As EcR is expressed in the same way in all lamina neurons, how is the cell-type specificity

147 of wiring genes determined? Interestingly, genes specifically expressed in L3, including Netrin,

148 are enriched for targets of the L3-specific TF Erm identified in a previous study ${ }^{12}$ (Hypergeometric

149 test, p-value $=2.7$ X 10-18; Fig. 3e; Extended data Fig. 17). Erm is expressed selectively in L3 early

150 in lamina development and remains expressed into the adult ${ }^{12,32}$ (Extended data Fig. 1c). Thus,

151 here the EcR pathway confers temporal specificity to a continuously expressed cell-type-specific

152 TF to ensure the precise spatial and temporal expression of wiring genes.

\section{Stepwise assembly of $L 5$ connectivity}

We next sought to assess whether the EcR cascade regulates sequential steps in wiring. For

155 this we turned to L5. L5 axon morphology in the medulla forms in a stepwise manner ${ }^{33}$ (Fig. 4b).

156 L5 targets to M5 and then arborizes in M1 and M2 (M1/2) (Fig. 1a, 4b). Arborization occurs in

157 two steps: 1) initial branching in M1 (between 48hAPF and 60hAPF) and, 2) growth from M1 to

158 M2 (between 75hAPF and 90hAPF) (Fig. 4b). EcR ${ }^{\mathrm{DN}}$ or EcR RNAi expression blocks step 1, while

159 Hr3 and ftz-f1 RNAi each blocks step 2 (Fig. 4a, b). These findings are consistent with the notion

160 that EcR regulates the expression of genes that promote extension of dendrites into M1 and that

161 Hr3 and Ftz-f1 execute a subsequent developmental step, some 24 hours later (Fig. 4b). Hr3

162 directly regulates $f t z-f 1$ expression ${ }^{17}$ (see Fig. 2a, Extended data Fig. 10d). Thus, Ftz-f1 may act

163 alone or in combination with $\mathrm{Hr} 3$, to regulate the expression of a set of genes necessary to control

164 targeting of dendrites to M2. As L5 makes synaptic connections with different partners in M1 and

$165 \mathrm{M} 2^{34}$, EcR may control cell surface proteins regulating synaptic specificity in M1, whereas

166 synaptic specificity in M2 may be controlled by cell recognition proteins regulated by Hr3, Ftz-f1, 167 or both. 
The phenotypes resulting from Hr4 RNAi are qualitatively different from knocking down M2 to M5 (Fig. 4a). Hr4 may regulate a stop signal, preventing extension beyond M2 to deeper

171 layers. Alternatively, as $\mathrm{Hr} 4$ is expressed at a much earlier step in development (see Fig. 2a), it

172 may repress a growth pathway acting in the initial extension into M1. As knock down experiments

173 of EcR pathway components led to marked changes in the expression of genes encoding different

174 cell surface and secreted proteins implicated in wiring (Fig. 4c, Supplementary Table 12), these or

175 discrete combinations of them, may function at different steps or in specific spatial domains of L5

176 axons to establish specific patterns of synaptic connections.

\section{Discussion}

Wiring the brain requires precise cell-type and temporally restricted expression of genes

179 encoding cell surface proteins ${ }^{2,3,12,13,35}$. Our studies uncovered a strategy that plays a widespread,

180 yet specific role, in regulating this process. We describe a genetic program expressed in most, if

181 not all, visual system neurons that controls the precise temporal patterns of expression of wiring 182 genes (Fig. 4d). These have cell-type specific outputs which reflect the integration of a shared 183 temporal program with diverse pathways controlling the development of different cell types. We

184 show that this temporal program regulates discrete steps in wiring specificity. cascade by the steroid hormone Ecdysone, comprising multiple transcription factors (such as EcR,

187 Hr3 and Ftz-f1) including members of the nuclear receptor family. These produce sequential waves 188 of gene expression ${ }^{16}$ (Fig. 2, 4d) which regulate different steps in wiring in different neurons. The 189 control of a cell-type-specific set of wiring genes is made possible, at least in part, by a 
combinatorial mechanism in which many members of this transcriptional cascade work with

191 different cell-type-specific TFs to produce different outputs ${ }^{36-38}$. Mechanisms that establish

192 cellular transcriptional landscapes early in development have been identified ${ }^{39-41}$, including cell-

193 type-specific TFs (e.g. Erm). These are expressed in postmitotic neurons and determine multiple

194 cell-type-dependent features, including transcription of genes required for circuit formation ${ }^{4,42,43}$.

195 By extrapolation of the dual control of Netrin by Erm and EcR in L3, we speculate that it is the 196 coordinate activities of these two classes of TFs that regulates developmental programs specifying

197 connectivity.

Dynamic expression of wiring genes, including cell-recognition molecules, poses an additional challenge for gene expression control. How are the transcriptomes of diverse neurontypes coordinated to ensure proper wiring? A global temporal signal, such as Ecdysone, is a possible mechanism to coordinate the development of multiple neuron types. For instance, the

202 Ecdysone-dependent timing of Netrin expression in L3 growth cones matches the arrival of R8

203 growth cones in M3 (Fig. 3d; Extended data Fig. 16a). Further experiments will be necessary to

204 determine whether Ecdysone not only regulates the timing of developmental steps, but also ensures

205 that these are coordinated between different neuron-types in the complex choreography leading to

206 circuit assembly.

207 Our studies rest on decades of genetic and biochemical experiments establishing the

208 Ecdysone-pathway as a temporal regulator of major developmental transitions, including larval

209 molts and metamorphosis, as well as controlling sequential divisions of postembryonic neuroblasts

210 and neuronal re-modelling ${ }^{16,23,37,38,44}$. Here we show that this program has been co-opted for the

211 temporal regulation of differentiation, and the coordinate development of different neuron types

212 leading to the complex circuitry of the fly visual system. The ability of the EcR cascade to function 
213 as a flexible temporal regulatory module used in multiple developmental contexts may be similar

214 to modules regulating spatial patterning (e.g. Hox genes and BMP/Wnt/Hh pathways ${ }^{45-47}$ ) which

215 act in context-dependent ways to generate different morphological outcomes. These findings raise

216 the intriguing possibility that similar timing mechanisms may also regulate the sequence of events

217 regulating wiring in other systems ${ }^{48}$ including the maturational sequence in the assembly of neural

218 circuits in the mammalian brain ${ }^{49}$. 
a

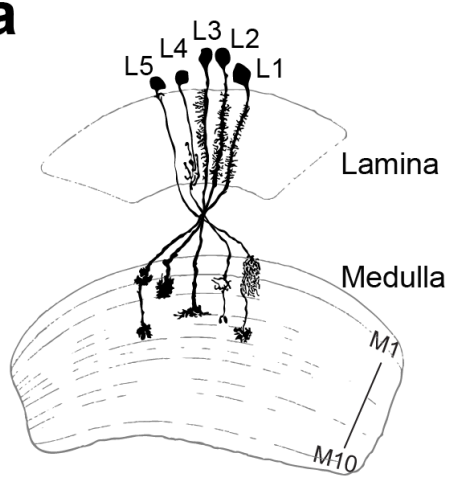

b Scheme for developmental transcriptomic analysis

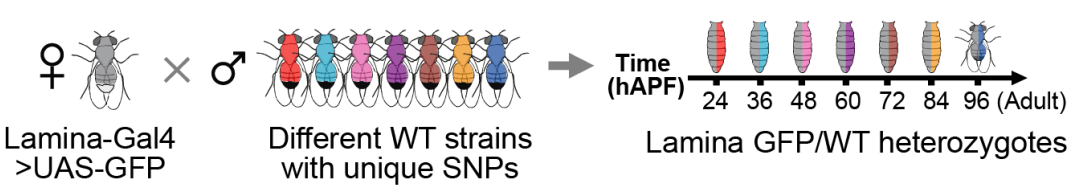

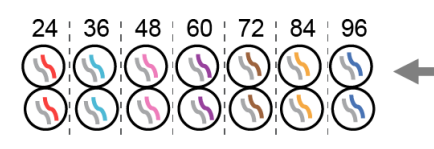

Demultiplex by SNPs

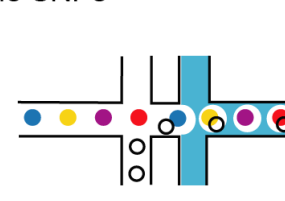

scRNA-seq

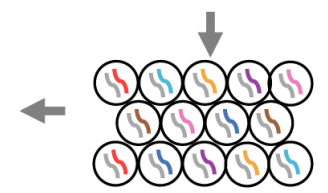

Multiplex \& FACS to purify lamina neurons

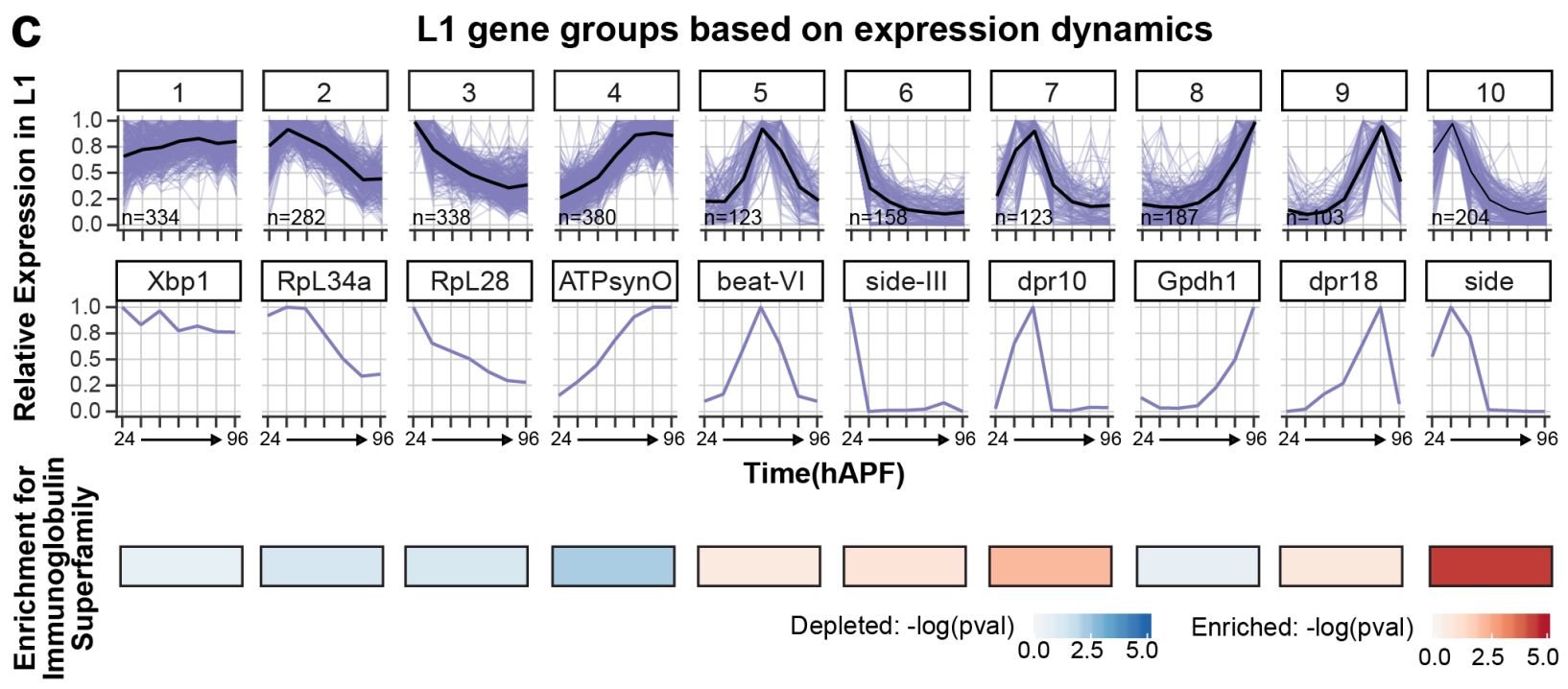

Fig. 1 | A developmental transcriptome reveals dynamic expression of wiring genes. a, Lamina neurons (L1-L5) in the Drosophila visual system project axons into the medulla. b, Scheme for single-cell RNA-Seq of lamina neurons (see Methods) using wildtype strains (DGRP) with unique SNPs as bar codes. Each time point includes lamina neurons from three animals, each heterozygous for a different SNP bar code. In total 21 different DGRP lines were used in this experiment. Thus, the transcripts of cells from each animal and their developmental stage (hours after pupal formation (hAPF)) were identified by unique SNPs. c, Gene groups generated via kmeans clustering using expression dynamics in L1 neurons (see Methods; for other lamina neurons see Extended data Fig. 3). Relative expression over development for each gene in the group is 
bioRxiv preprint doi: https://doi.org/10.1101/2020.09.18.304410; this version posted September 19,2020 . The copyright holder for this preprint (which was not certified by peer review) is the author/funder, who has granted bioRxiv a license to display the preprint in perpetuity. It is made available under aCC-BY-NC-ND 4.0 International license.

229 group. A gene from each group is shown (e.g. Xbp1). Heat map depicts enrichment $\left(-\log _{10}\right.$ p-

230 values) of Immunoglobulin Superfamily genes over that expected by chance (see Methods). 
a
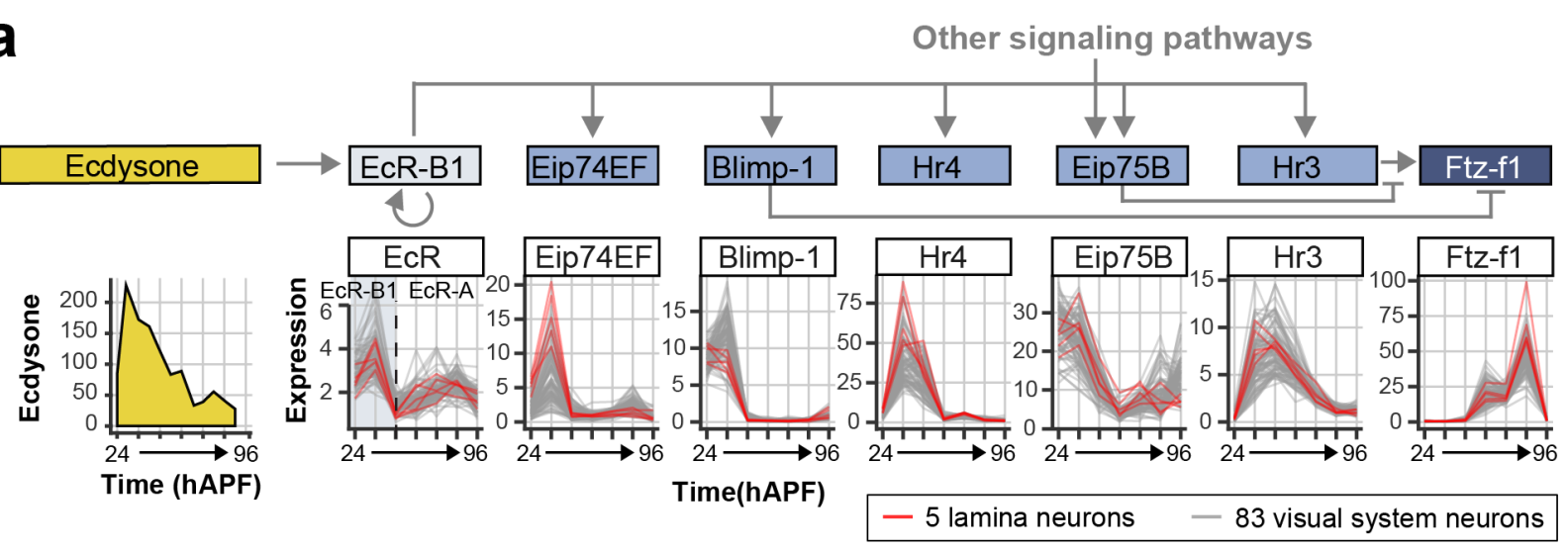

b wT:

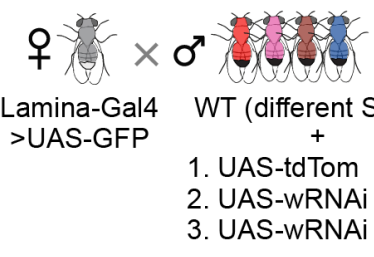

Mutant:

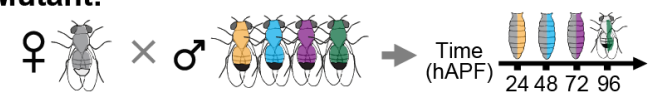

$\underset{\substack{\text { Lamina-Gal4 } \\>\text { WAS-GFP (different SNPs) }}+}{+}$

$>$ UAS-GFP
1. UAS-ECR

$$
\begin{aligned}
& \text { 2. UAS-EcR RNAi } \\
& \text { 3. UAS-Hr3 RNAi }
\end{aligned}
$$

C Expression of EcR ${ }^{\mathrm{DN}}$ affected genes in wildtype lamina neurons

\section{d Examples of wiring genes affected by EcR ${ }^{\mathrm{DN}}$}

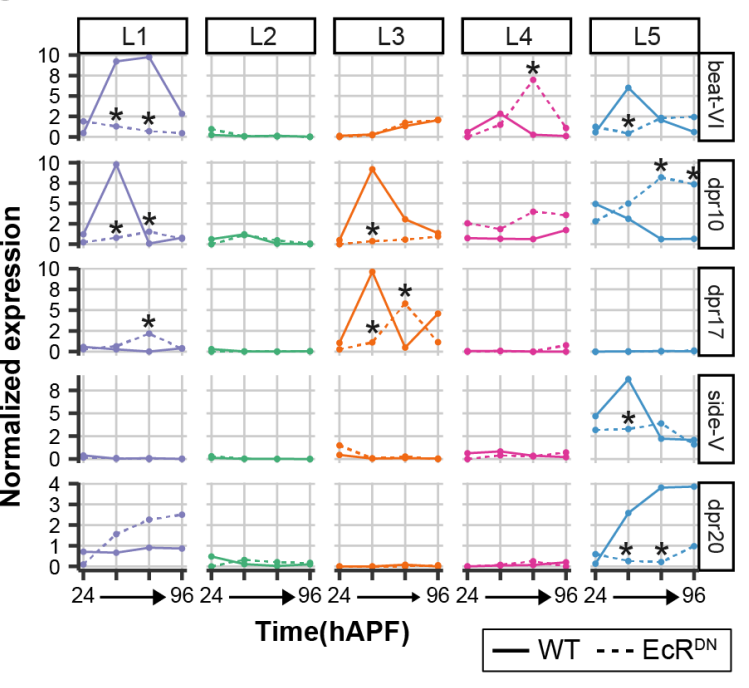

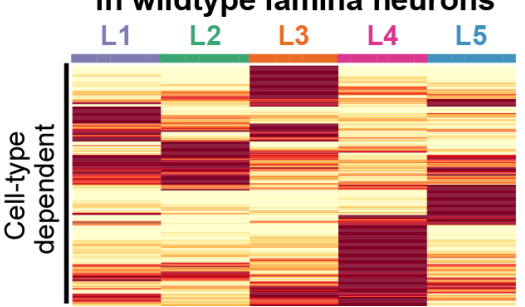

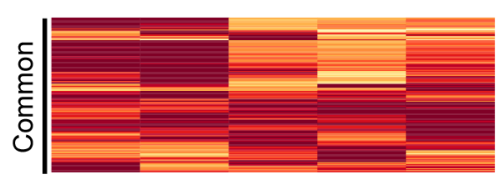

\begin{tabular}{|lll|}
\hline Relative expression & & \\
& 0 & 1 \\
\hline
\end{tabular}

e Examples of wiring genes affected by $\mathrm{Hr} 3 \mathrm{RNAi}$

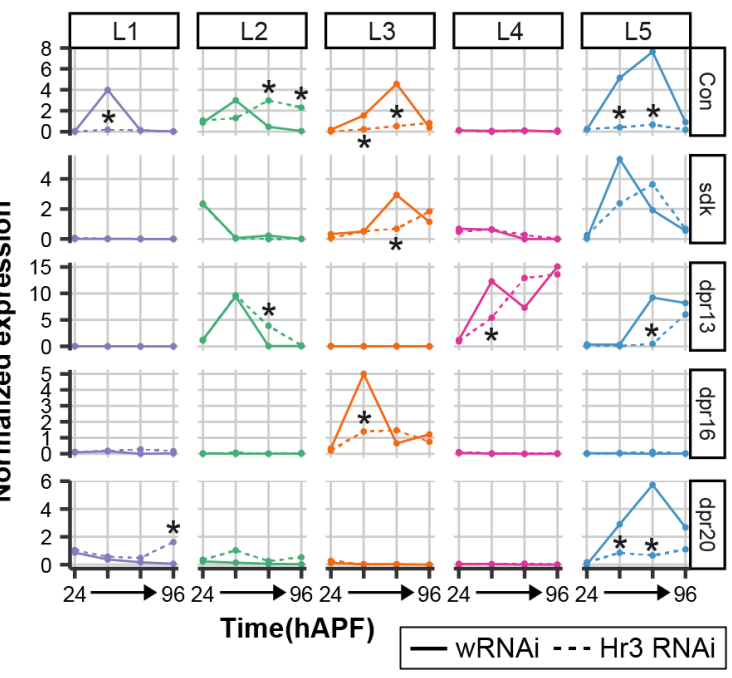

231 Fig. 2 | Wiring genes in different cell-types are controlled by an Ecdysone-induced 
233 the Ecdysone-induced transcriptional cascade. Only a subset of the known interactions is shown.

234 In the plots, red lines show normalized expression of these transcription factors in lamina neurons

235 and grey lines show expression dynamics in 83 other neuron-types (data from Kurmangaliyev et

236 al. revision under review) (bottom). Isoform change from EcR-B1 to EcR-A during development

237 is shown. Concentration of circulating Ecdysone over development is shown on the left (adapted

238 from Pak and Gilbert ${ }^{21}$ ) b, Scheme for scRNA-Seq-based analysis of genes affected by

239 perturbation of EcR or $\mathrm{Hr} 3$ activity in lamina neurons. Mutant and control brains for each

240 perturbation were analyzed together in three separate experiments. UAS-tdTom and UAS-wRNAi

241 were use as controls for $\mathrm{EcR}^{\mathrm{DN}}$ and EcR (and Hr3) RNAi, respectively. c, Heat map showing

242 relative expression in wildtype cells of genes affected by $E c R^{\mathrm{DN}}$ at $48 \mathrm{hAPF}$. Targets of EcR are

243 divided into two categories: Cell-type dependent, in any pairwise comparison fold difference $\geq 2$,

244 p-value $\leq$ 0.05, 303 genes; Common targets, all remaining EcR targets, 208 genes. d, Expression

245 of some wiring genes affected by $\mathrm{EcR}^{\mathrm{DN}}$. Solid line, expression in wildtype cells; Dashed line,

246 expression in EcR ${ }^{\mathrm{DN}}$ cells. e, Expression of some wiring genes altered by Hr3 RNAi. Solid line,

247 expression in wildtype cells; Dashed line, expression in Hr3 RNAi cells; *, difference in

248 expression between wildtype and perturbation $\geq 2$-fold, $p$-value $\leq 0.05$. 
a

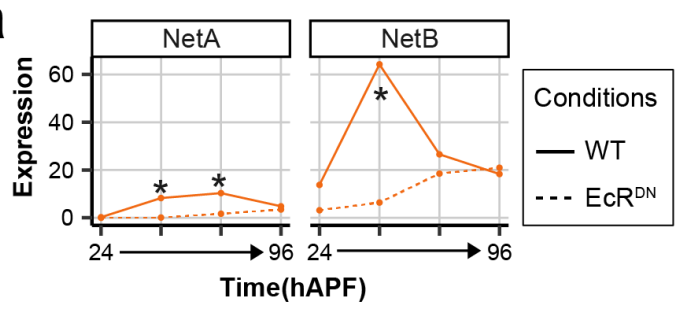

b
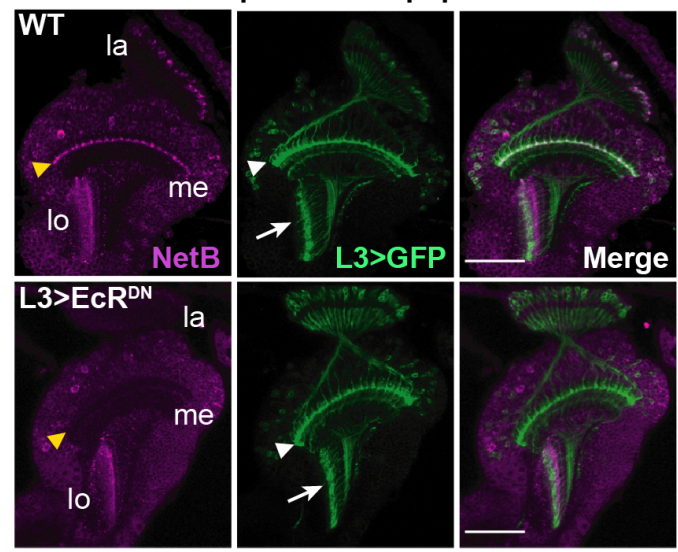

d

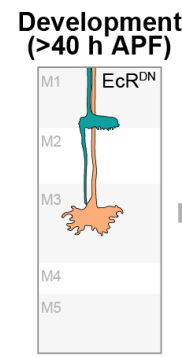

40hAPF
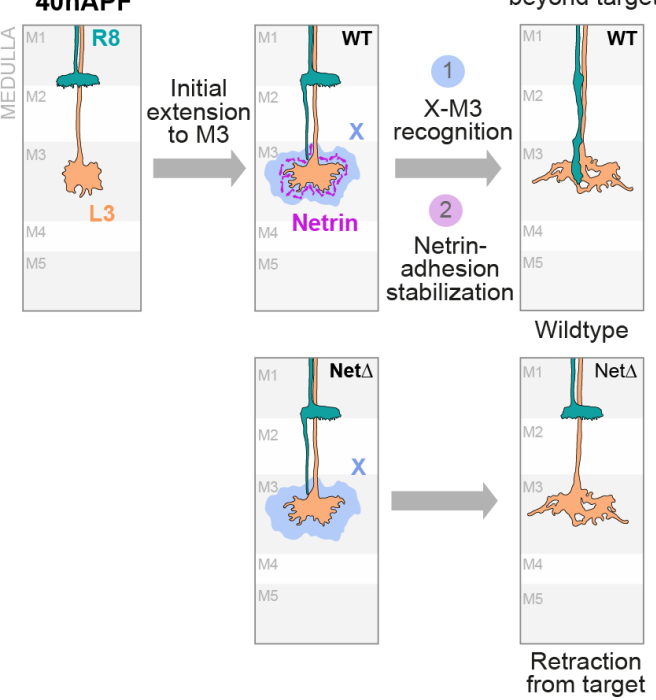

C R8 targeting in medulla
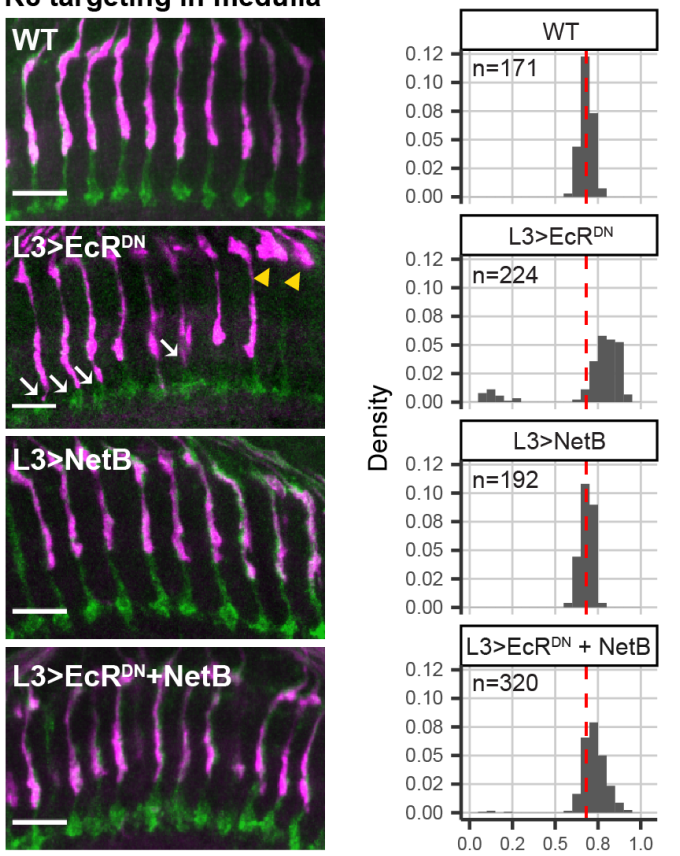

R8 24B10

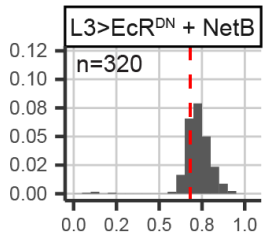

R8:M6 depth ratio

e Expression of L3-specific EcR-target genes

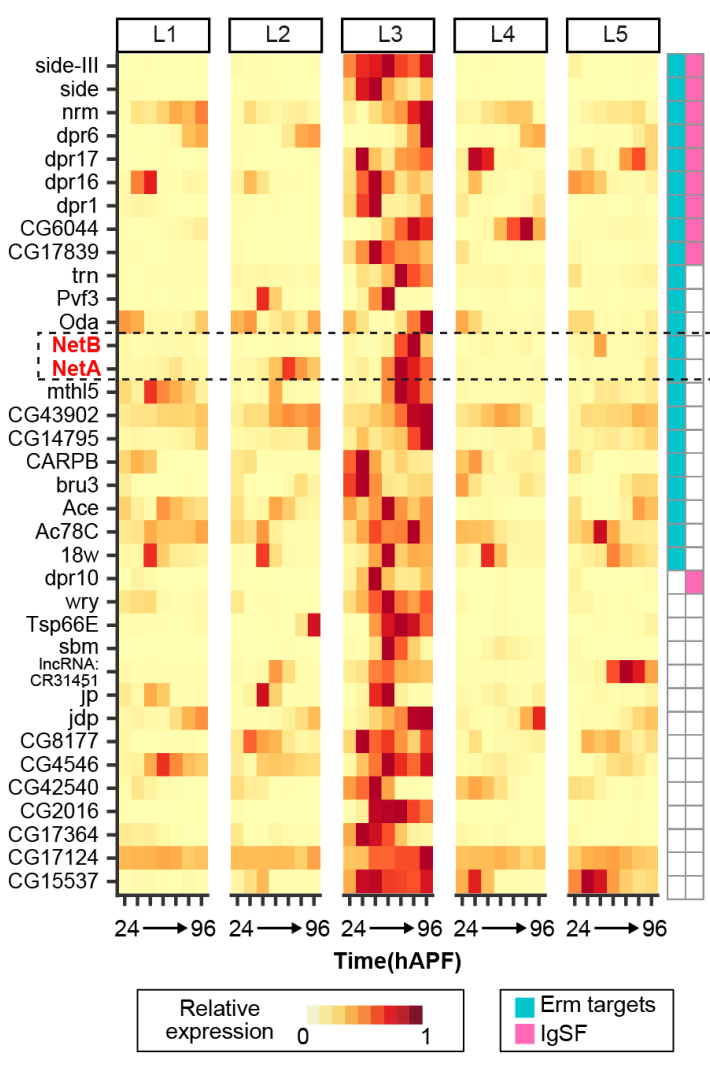

249 Fig. 3 | EcR-dependent program in L3 neurons controls R8 targeting. a, Normalized 
$251 \mathrm{EcR}^{\mathrm{DN}}$ (dashed lines) expressing L3 neurons. *, difference in expression between wildtype and

$252 \quad \mathrm{EcR}^{\mathrm{DN}} \geq 2$-fold, $\mathrm{p}$-value $<0.05$. b, Anti-NetB staining (magenta) at $48 \mathrm{hAPF} \pm \mathrm{EcR}^{\mathrm{DN}}$ expression

253 in L3 neurons (green). Netrin expression in M3 is lost in response to EcR ${ }^{\mathrm{DN}}$. Note, as an internal

254 control, that NetB expression in the lobula (lo) is not affected. la, lamina; me, medulla; yellow

255 arrowhead, M3 layer; white arrowheads, L3 growth cones (some other cell types with axons in the

256 lobula are also labeled (white arrows)). Scale bar, $50 \mu \mathrm{m}$. c, Morphology of R8 axons (magenta)

257 with wildtype L3 neurons (top). Other panels from top to bottom show L3s expressing EcR ${ }^{\mathrm{DN}}$,

258 NetB and both EcR ${ }^{\mathrm{DN}}$ and NetB. Left panels: R8 axons extending beyond M3 (white arrows); R8

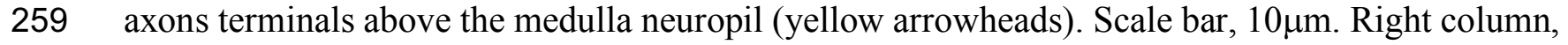

260 distribution of R8 axon depths represented as ratio of depth of R8 to M6 (medulla layer 6 indicated

261 in green in the left panels). Red line, median of the distribution in wildtype. Distribution with WT

262 and $\mathrm{EcR}^{\mathrm{DN}}$ expressing L3 is significantly different (Kolmogorov-Smirnov test, $\mathrm{p}$-value $<10^{-15}$, see

263 Extended data Fig. 16c for other comparisons). n, number of neurons observed (6-10 animals for

264 all conditions). d, Model for control of R8 targeting by L3. EcR controls expression of Netrin and

265 one or more other molecules (X) in L3. Netrin is required for adhesion of R8 growth cones to

266 processes in $\mathrm{M} 3$ and $\mathrm{X}$ is required for recognition of $\mathrm{M} 3$ by $\mathrm{R} 8$ growth cones to terminate

267 extension ${ }^{30}$. e, Heat map showing relative expression of L3-specific targets of EcR (see Methods).

268 Aqua, targets of transcription factor Erm identified in Peng et al. ${ }^{32}$; Magenta, Immunoglobulin

269 Superfamily genes; Dashed lines, NetA and NetB. 
a

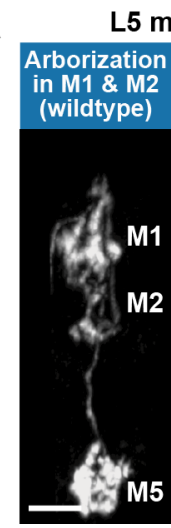

WT

b

Stepwise morphogenesis of L5

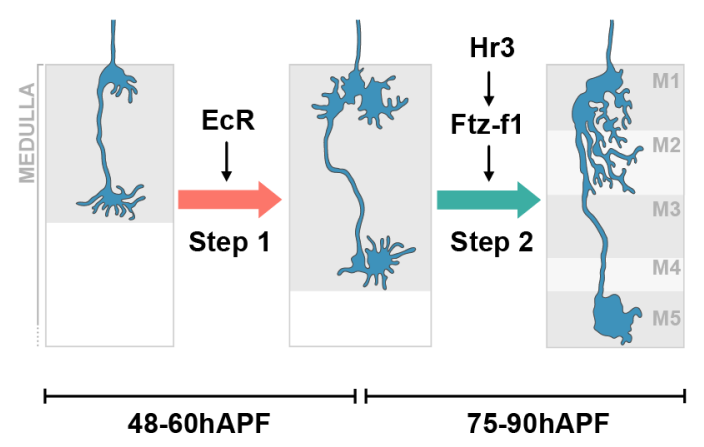

d

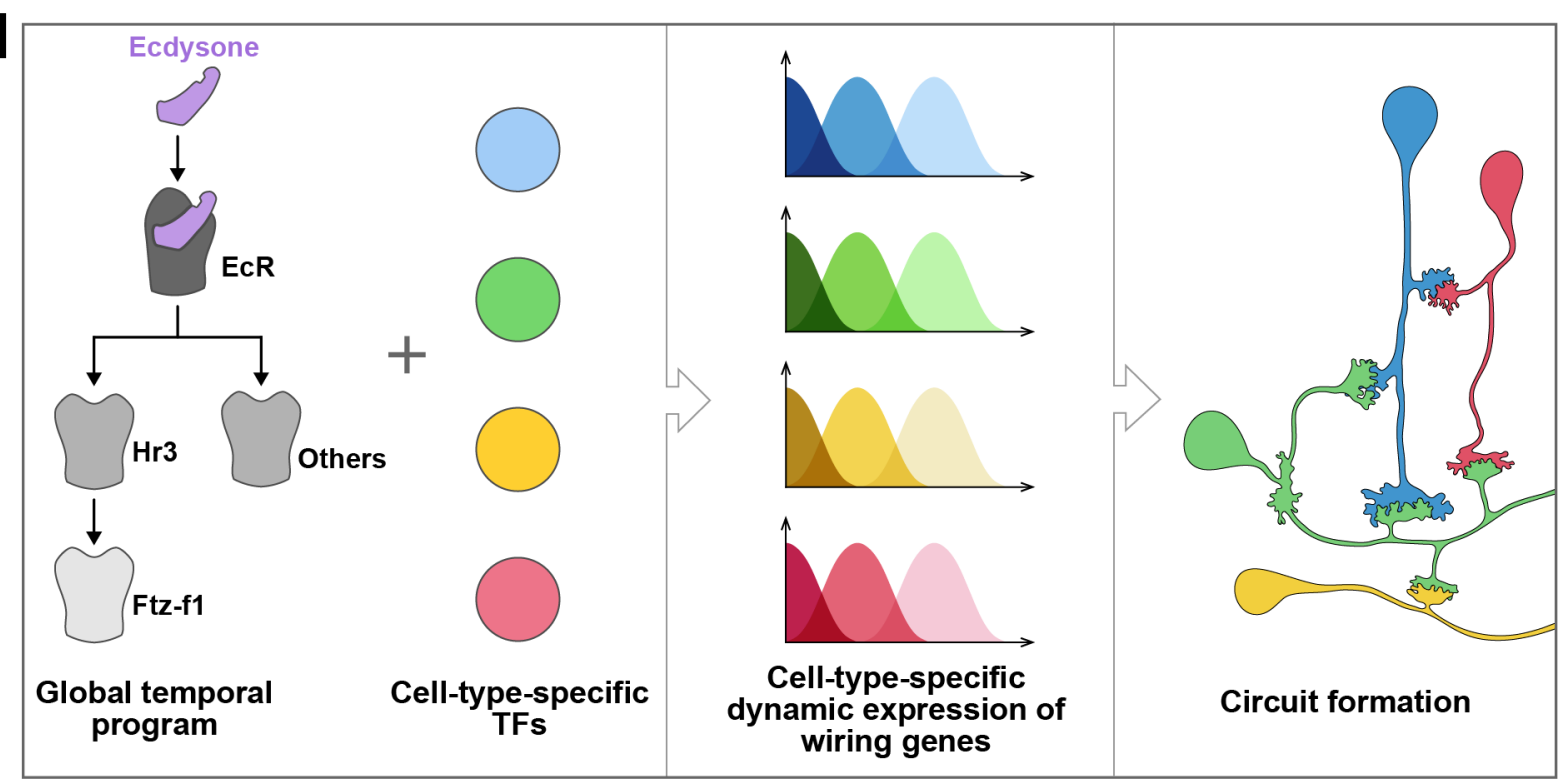

270 Fig. 4 | The EcR cascade controls the stepwise arborization of L5 axons. a, L5 axonal

271 arborization defects (left) and their distributions in wildtype L5's, or under pan-lamina expression

272 of EcR ${ }^{\text {DN }}$, EcR RNAi, Hr3 RNAi, Hr4 RNAi or ftz-f1 RNAi (right). *, Fisher's exact test p-value 
$273<0.01 . \mathrm{n}$, number of neurons observed (4 -13 animals for all conditions). In wildtype only the axon

274 extends to M5; L5 neurons in Hr4 RNAi animals extend an additional process to M5. Scale bars,

$2755 \mu \mathrm{m} . \mathbf{b}$, Schematic of the stepwise morphogenesis of L5 in the medulla ${ }^{33}$ showing the expected

276 roles for EcR, Hr3 and Ftz-f1 based on data in panel a) and previous biochemical and gene

277 expression studies (see Fig. 2a). The role of $\mathrm{Hr} 4$ in this process is unclear (see text). c, Volcano

278 plots showing candidate genes involved in step1 (Left, 131 genes, significantly affected in EcR ${ }^{\mathrm{DN}}$

279 at $48 \mathrm{hAPF}$ and not affected in Hr3 RNAi at any timepoint) and step2 (Right, 124 genes,

280 significantly affected in $\mathrm{Hr} 3 \mathrm{RNAi}$ at $72 \mathrm{hAPF}$, regardless of effect of $\mathrm{EcR}^{\mathrm{DN}}$ ) of L5 arborization.

281 Immunoglobulin Superfamily genes are highlighted. d, Model: Control of wiring genes through a

282 global temporal program acting in combination with cell-type-specific transcription factors (see

283 Discussion). Other temporal regulators may also contribute to wiring. 


\section{References}

285 1. Südhof, T. C. Towards an Understanding of Synapse Formation. Neuron 100, 276--293

286 (2018).

287 2. Hassan, B. A. \& Hiesinger, P. R. Beyond Molecular Codes: Simple Rules to Wire Complex

288 Brains. Cell 163, 285--291 (2015).

289 3. Yogev, S. \& Shen, K. Cellular and Molecular Mechanisms of Synaptic Specificity. Annu Rev

290 Cell Dev Bi 30, 417--437 (2014).

291 4. Hobert, O. Chapter Twenty-Five - Terminal Selectors of Neuronal Identity. in Current Topics

292 in Developmental Biology (ed. Wassarman, P. M.) vol. 116 455--475 (Academic Press, 2016).

293 5. Hong, W. \& Luo, L. Genetic Control of Wiring Specificity in the Fly Olfactory System.

294 Genetics 196, 17--29 (2014).

295 6. Dasen, J. S. \& Jessell, T. M. Chapter Six Hox Networks and the Origins of Motor Neuron

296 Diversity. in Current Topics in Developmental Biology vol. 88 169--200 (Academic Press, 297 2009).

298 7. Zipursky, S. L. \& Sanes, J. R. Chemoaffinity Revisited: Dscams, Protocadherins, and Neural 299 Circuit Assembly. Cell 143, 343--353 (2010).

300 8. Sanes, J. R. \& Zipursky, S. L. Design Principles of Insect and Vertebrate Visual Systems.

301 Neuron 66, 15--36 (2010).

302 9. Pecot, M. Y. et al. Sequential Axon-Derived Signals Couple Target Survival and Layer

303 Specificity in the Drosophila Visual System. Neuron 82, 320--333 (2014).

304 10. Chen, Y. et al. Cell-type-Specific Labeling of Synapses In Vivo through Synaptic Tagging

305 with Recombination. Neuron 81, 280--293 (2014). 
306 11. Mackay, T. F. C. et al. The extitDrosophila melanogaster Genetic Reference Panel. Nature

$307 \quad 482,173--178(2012)$.

308 12. Tan, L. et al. Ig Superfamily Ligand and Receptor Pairs Expressed in Synaptic Partners in

309 Drosophila. Cell 163, 1756--1769 (2015).

310 13. Li, H. et al. Classifying Drosophila Olfactory Projection Neuron Subtypes by Single-Cell

311 RNA Sequencing. Cell 171, 1206--1220.e22 (2017).

312 14. Favuzzi, E. et al. Distinct molecular programs regulate synapse specificity in cortical

313 inhibitory circuits. Sci New York N Y 363, 413--417 (2019).

314 15. Jassal, B. et al. The reactome pathway knowledgebase. Nucleic Acids Res 48, D498--D503

315 (2020).

316 16. Riddiford, L. M., Cherbas, P. \& Truman, J. W. Ecdysone receptors and their biological

317 actions. Vitamins Hormones 60, 1--73 (2000).

318 17. White, K. P., Hurban, P., Watanabe, T. \& Hogness, D. S. Coordination of Drosophila

319 metamorphosis by two ecdysone-induced nuclear receptors. Sci New York N Y 276, 114--117

320 (1997).

321 18. Truman, J. W., Talbot, W. S., Fahrbach, S. E. \& Hogness, D. S. Ecdysone receptor

322 expression in the CNS correlates with stage-specific responses to ecdysteroids during Drosophila

323 and Manduca development. Dev Camb Engl 120, 219--234 (1994).

324 19. Agawa, Y. et al. Drosophila Blimp-1 Is a Transient Transcriptional Repressor That Controls

325 Timing of the Ecdysone-Induced Developmental Pathway. Mol Cell Biol 27, 8739--8747 (2007).

326 20. Rabinovich, D., Yaniv, S. P., Alyagor, I. \& Schuldiner, O. Nitric Oxide as a Switching

327 Mechanism between Axon Degeneration and Regrowth during Developmental Remodeling. Cell

328 164, 170--182 (2016). 
21. Pak, M. D. \& Gilbert, L. I. A Developmental Analysis of Ecdysteroids During the

331 22. Boulanger, A. \& Dura, J.-M. Nuclear receptors and Drosophila neuronal remodeling.

332 Biochimica Et Biophysica Acta Bba - Gene Regul Mech 1849, 187--195 (2015).

333 23. Alyagor, I. et al. Combining Developmental and Perturbation-Seq Uncovers Transcriptional

334 Modules Orchestrating Neuronal Remodeling. Dev Cell 47, 38--52.e6 (2018).

335 24. Imrichová, H., Hulselmans, G., Atak, Z. K., Potier, D. \& Aerts, S. i-cisTarget 2015 update:

336 generalized cis-regulatory enrichment analysis in human, mouse and fly. Nucleic Acids Res 43,

337 W57--W64 (2015).

338 25. Buenrostro, J. D., Wu, B., Chang, H. Y. \& Greenleaf, W. J. ATAC-seq: A Method for

339 Assaying Chromatin Accessibility Genome-Wide. Curr Protoc Mol Biology Ed Frederick M

340 Ausubel Et Al 109, 21.29.1--9 (2015).

341 26. Olivares, A. M., Moreno-Ramos, O. A. \& Haider, N. B. Role of Nuclear Receptors in Central

342 Nervous System Development and Associated Diseases. J Exp Neurosci 9, 93--121 (2016).

343 27. Cherbas, L., Hu, X., Zhimulev, I., Belyaeva, E. \& Cherbas, P. EcR isoforms in Drosophila:

344 testing tissue-specific requirements by targeted blockade and rescue. Development 130, 271--284

345 (2003).

346 28. Özel, M. N., Langen, M., Hassan, B. A. \& Hiesinger, P. R. Filopodial dynamics and growth

347 cone stabilization in Drosophila visual circuit development. Elife 4, e10721 (2015).

348 29. Timofeev, K., Joly, W., Hadjieconomou, D. \& Salecker, I. Localized Netrins Act as

349 Positional Cues to Control Layer-Specific Targeting of Photoreceptor Axons in Drosophila.

350 Neuron 75, 80--93 (2012). 
351 30. Akin, O. \& Zipursky, S. L. Frazzled promotes growth cone attachment at the source of a

352 Netrin gradient in the Drosophila visual system. Elife 5, e20762 (2016).

353 31. Xu, C. et al. Control of Synaptic Specificity by Establishing a Relative Preference for

354 Synaptic Partners. Neuron 103, 865--877.e7 (2019).

355 32. Peng, J. et al. Drosophila Fezf coordinates laminar-specific connectivity through cell-

356 intrinsic and cell-extrinsic mechanisms. Elife 7, (2018).

357 33. Nern, A., Zhu, Y. \& Zipursky, S. L. Local N-Cadherin Interactions Mediate Distinct Steps in

358 the Targeting of Lamina Neurons. Neuron 58, 34--41 (2008).

359 34. Takemura, S. et al. A visual motion detection circuit suggested by extitDrosophila

360 connectomics. Nature 500, 175--181 (2013).

361 35. Kurmangaliyev, Y. Z., Yoo, J., LoCascio, S. A. \& Zipursky, S. L. Modular transcriptional

362 programs separately define axon and dendrite connectivity. Elife 8, e50822 (2019).

363 36. Barolo, S. \& Posakony, J. W. Three habits of highly effective signaling pathways: principles

364 of transcriptional control by developmental cell signaling. Gene Dev 16, 1167--1181 (2002).

365 37. Shlyueva, D. et al. Hormone-responsive enhancer-activity maps reveal predictive motifs,

366 indirect repression, and targeting of closed chromatin. Mol Cell 54, 180--192 (2014).

367 38. Uyehara, C. M. \& McKay, D. J. Direct and widespread role for the nuclear receptor EcR in

368 mediating the response to ecdysone in Drosophila. Proc National Acad Sci 116, 9893--9902

369 (2019).

370 39. Erclik, T. et al. Integration of temporal and spatial patterning generates neural diversity.

$371 \quad$ Nature 541, 365--370 (2017).

372 40. McDonald, J. A. et al. Dorsoventral patterning in the Drosophila central nervous system: the 373 vnd homeobox gene specifies ventral column identity. Gene Dev 12, 3603--3612 (1998). 
374 41. Cochella, L. \& Hobert, O. Embryonic Priming of a miRNA Locus Predetermines Postmitotic

375 Neuronal Left/Right Asymmetry in C. elegans. Cell 151, 1229--1242 (2012).

376 42. Kratsios, P. et al. Transcriptional Coordination of Synaptogenesis and Neurotransmitter

377 Signaling. Curr Biol 25, 1282--1295 (2015).

378 43. Philbrook, A. et al. Neurexin directs partner-specific synaptic connectivity in C. elegans.

379 Elife 7, e35692 (2018).

380 44. Syed, M. H., Mark, B. \& Doe, C. Q. Steroid hormone induction of temporal gene expression

381 in Drosophila brain neuroblasts generates neuronal and glial diversity. Elife 6, e26287 (2017).

382 45. Altmann, C. R. \& Brivanlou, A. H. Neural patterning in the vertebrate embryo. Int Rev Cytol

$383203,447-482(2001)$.

384 46. Briscoe, J. \& Small, S. Morphogen rules: design principles of gradient-mediated embryo

385 patterning. Development 142, 3996-4009 (2015).

386 47. Gaunt, S. J. Hox cluster genes and collinearities throughout the tree of animal life. Int J Dev

387 Biol 62, 673-683 (2018).

388 48. Lawson, H. et al. The Makorin lep-2 and the lncRNA lep-5 regulate lin-28 to schedule sexual

389 maturation of the C. elegans nervous system. Elife 8, (2019).

390 49. McCarthy, M. M. Estradiol and the Developing Brain. Physiol Rev 88, 91--124 (2008). 
Methods

Fly husbandry and stocks

Maintenance and rearing of fly lines, as well as staging of pupae was done as described in

394 Tan et al. ${ }^{12}$ Fly lines used in this work are listed in Supplementary Table 1.

\section{Multiplexed single cell transcriptomic analysis}

For transcriptomic analysis of developing wildtype lamina neurons, w; UAS-H2A-GFP; 9B08-Gal4/Tm6B, tb females were crossed with males from different DGRP backgrounds (wildtype, see Supplementary Table 1 for list of DGRPs used). F1 prepupae were staged as in Kurmangaliyev et al. ${ }^{35}$ Males were only included in the analysis if no significant differences in gene expression were found with female pupae of the same genotype and developmental time point. Pupae corresponding to different developmental stages (see Fig. 1b) were all dissected, dissociated and processed at the same time. Each developmental time point was represented by $\geq$ 2 DGRP heterozygotes, and only one animal/ DGRP heterozygote was dissected. Tissue dissociation, FACS and preparation of single-cell libraries using 10X Genomics Chromium (v3) were carried out similar to Kurmangaliyev et al, except that H2A-GFP expression was used to enrich for lamina neurons (see Extended data Fig. 1a). All libraries were sequenced on a NextSeq500 platform (single-end 75bp).

For transcriptomic analysis involving UAS EcR ${ }^{\mathrm{DN}}$ (BDSC \#6872), males carrying a single DGRP autonomous chromosome - w; $\left(\mathrm{UAS} \mathrm{EcR}^{\mathrm{DN}}\right.$ or UAS tdTom); DGRP/ Tm6b, tb were crossed with w; UAS-H2A-GFP; 9B08-Gal4/Tm6B females. For experiments involving UAS EcR RNAi (BDSC \#9326) or UAS Hr3 RNAi (BDSC \#27253), w; DGRP; (UAS wRNAi or UAS EcR RNAi or UAS Hr3 RNAi)/ Tm6B, tb males were crossed with UAS Dcr2; UAS H2A-GFP; 9B08 
413 Gal4/ Tm6B, tb females (see Fig. 2b and Supplementary Table 1). UAS tdTom and UAS wRNAi

414 were used as controls.

\section{5 scRNA-seq data pre-processing}

416 Raw fastq read files were processed using Cell Ranger (3.1.0) with default parameters.

417 Seurat V3 was used for all preliminary analyses. FlyBase reference genome (release $6.29^{50}$ ) was

418 used for alignment and annotation. Profiled single cells were identified as coming from a particular

419 developmental time-point and genetic background based on the natural genomic variance within

420 the different parent DGRP lines. This was done using the pipeline described in Kurmangalyev et

421 al., with the following modifications: the count of minor allele that equals to 2 among the analyzed

422 DGRP strains were used as genomic variants to assign the profiled single cells to different DGRP

423 parent lines. Only single cells with: 1) number of genes between 200 and 3000, 2) number of

424 mitochondrial transcripts $<20 \%$ of all transcripts, and 3) assignment to a unique DGRP parent

425 line; were used for all downstream analyses.

426 To identify different lamina neuron subtypes, all cells from a particular experiment were

427 integrated as described in Seurat v3 workflow and then subjected to unsupervised clustering, thus

428 disregarding global temporal gene expression changes (similar to Kurmangaliyev et al.).

429 Previously identified lamina neuron subtype specific genes ${ }^{12}$ were used to assign each cluster to a

430 cell-type. Cells not assigned to a lamina neuron-type cluster were removed from subsequent

431 analyses. Average expression for each gene for each cell-type at a particular timepoint and genetic

432 background was calculated using normalized expression values prior to integration. 


\section{Bulk RNA-seq}

Bulk RNA-Seq analysis of L1 neurons at 40hAPF, 60hAPF and 72hAPF was performed as in Tan et al. ${ }^{12}$ At least 2 replicates were generated for each timepoint. A minimum of 2000 cells

436 were sorted for each experiment. cDNA libraries were created using the SMART-Seq2 protocol $^{51}$,

437 which were then sequenced together using the HiSeq4000 platform (paired-end 50bp).

438 Raw fastq reads files were mapped to FlyBase reference genome (release 6.29) using STaR

439 (2.6.0) and only uniquely mapped reads were collected. Genes with counts per million (CPM) $\geq 4$

440 in more than 2 samples were used for normalization with R package edgeR (3.26.8). Gene

441 expression was quantified using RPKM units (Reads Per Kilobase of exon per Million reads

442 mapped), calculated based on reads in the sum of exons using customized scripts. The correlation

443 between biological replicates were calculated using Spearman correlation for top 500 genes with

444 the highest variation across all samples.

\section{Bulk ATAC- seq}

446 At least $8000 \mathrm{L1}$ neurons were FACS purified at 40hAPF, 60hAPF and $72 \mathrm{hAPF}$ as

447 described in Tan et al. ${ }^{12}$ Biological duplicates were generated for each timepoint. ATAC-Seq

448 libraries were generated as per the following protocol, which was modified from the one described 449 in Buenrostro et al. ${ }^{52}$ : 1) FACS purified cells were collected in $300 \mathrm{ul} 1 \mathrm{X}$ PBS and spun at $800 \mathrm{xg}$

450 for $8^{\prime}$ at $4^{\circ} \mathrm{C}$. 2) Cell pellet was then directly resuspended in $50 \mu 1$ of a tagmentation enzyme mix

$451(25 \mu 1$ Nextera DNA library prep kit - Tagment DNA Buffer, $19 \mu 1$ Nuclease-free water, $5 \mu 11 \%$

452 IGEPAL CA630, $1 \mu 1$ Nextera DNA library prep kit - Tagment DNA Enzyme) and incubated at $45337^{\circ} \mathrm{C}$ for $30^{\prime}$ with constant agitation at 400rpm. 3) DNA was purified from the tagmentation mixes

454 using the Qiagen minElute Reaction Cleanup Kit as per the manufacturer's protocol. 4) A 10 $\mu 1$ 
455 qPCR reaction was setup to determine the optimum number of amplification cycles (similar to

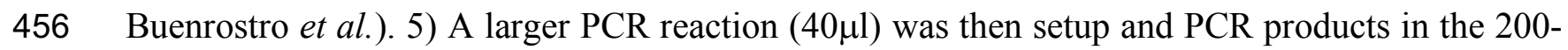

$457500 \mathrm{bp}$ range were gel purified. 6) Steps 4 and 5 were repeated, this time with barcoded primers

458 (see Buenrostro et al.) to allow multiplexing of all libraries onto a single lane of HiSeq4000

459 (paired-end 50bp).

460 Raw fastq reads files were mapped to FlyBase reference genome (release 6.29) using

461 Bowtie2 (2.2.9) and uniquely mapped genes were kept. All samples were pooled together prior to

462 peak calling. Read start positions were shifted +4 or $-5 \mathrm{bp}$ and used for peak calling using MACS2

463 (2.1.1) with parameters “-q 0.01 --nomodel --shift -100 --extsize 200”. There were 26,122 peaks

464 identified. Then bedtools multicov (2.27.1) was used to sum the total reads within each peak

465 separately for each sample. Peaks with $\mathrm{CPM} \geq 4$ were used for counts normalization with edgeR.

466 Correlations between biological replicates were calculated using Spearman correlation for the top

467500 peak regions with the highest variation of peak levels across all samples. Distribution of the

468 top 5000 peaks across different genomic features at 40hAPF, 60hAPF and $72 \mathrm{hAPF}$ was

469 determined using the R package ChIPseeker (1.20.0). Differential peak analysis was performed

470 using edgeR. Fold-change $\geq 2$ and adjusted p-value $\leq 0.05$ were chosen as the cutoffs to define

471 peaks with differential accessibility between time points. Each ATAC peak was associated to the

472 nearest gene based on proximity to the transcription start site using ChIPseeker. To compare

473 change of ATAC-seq peak accessibility over time and change in expression of nearest gene, $\ln$ (fold

474 change ATAC-seq peak read coverage) was plotted against $\ln$ (fold change expression of nearest

475 gene) for each ATAC-Seq peak (Extended data Fig. 6). Bulk RNA-Seq data was used for this, as

476 this transcriptome dataset had been generated for the same time points as for ATAC-Seq. This was 
477 done separately for peaks differentially accessible between 40hAPF and 60hAPF (Extended data

478 Fig. $6 \mathrm{~b}(\mathrm{left}))$ and between $60 \mathrm{hAPF}$ and 72hAPF (Extended data Fig. 6b (right)).

479 Comparison of scRNA-Seq with other datasets

480 For comparison of lamina neuron transcriptomes generated here with lamina neuron

481 transcriptomes generated in Kurmangaliyev et al., 500 genes with the highest variance across all

482 cell types and all timepoints were used to calculate Spearman correlation. Comparison of L1

483 transcriptome generated using scRNA-Seq and L1 transcriptome obtained from bulk RNA-Seq

484 was done similarly.

\section{$485 \quad$ k-means cluster analysis}

Prior to clustering, all average gene expression from scRNA-seq transcriptomic analysis

487 were normalized to maximum expression in each cell type across development. k-means clustering 488 was performed using kmeans function in $\mathrm{R}$ (centers $=10$ and nstart $=20)$.

\section{Analysis of gene group traits}

$490 \quad$ IgSF enrichment

491 A list of IgSF members was obtained from the InterPro database (IPR036179). Enrichment

492 within each gene group over expected by chance was then calculated using hypergeometric

493 distribution analysis. The set of genes expressed in a cell-type across development was taken as

494 the universal set $(\mathrm{N})$. Then number of genes in a group (n) and number of IgSFs expressed in a

495 cell-type (m) were used as the sample size and number of successes in population respectively.

496 Enrichment was calculated based on actual overlap between $\mathrm{n}$ and $\mathrm{m}$, and the expected overlap 
497 between the two, defined by $(\mathrm{n} \times \mathrm{m}) / \mathrm{N}$. For enrichment of IgSF members within cell-type

498 dependent targets of EcR, universal set was taken as all genes expressed in all lamina neurons over

499 development. Sample size and number of successes in the population were taken as: the total

500 number of IgSF genes expressed over development in all lamina neurons (n), and the total number

501 of cell-type dependent targets of EcR (m) respectively. Enrichment was calculated as described

502 above.

503 Gene Ontology enrichment analysis

504 Gene Ontology enrichment analysis for each gene group was performed using a R package

505 TopGO (2.36.0) using GO terms obtained from FlyBase (GO-gene association file version 2.1).

506 Weight algorithm and Fisher's exact test were used to calculate p-value. Categories that have p-

507 value $\leq 0.01$ were considered as enriched

508 Effect of perturbation of EcR and $\mathrm{Hr} 3$

509 For each perturbation, expression of each gene was normalized to maximum expression in

510 each cell type across development and genetic background. The normalized gene expression of all

511 genes was averaged separately for each genetic background to plot average behaviors for each

512 gene group (Extended data Fig. 11).

\section{Differential gene expression analysis}

514 Wilcoxon rank-sum test (fold change $\geq 2$, adjusted $p$-value $\leq 0.05$ ) was used to identify

515 genes differentially expressed between timepoints, cell-types or as a consequence of EcR/ $\mathrm{Hr} 3$

516 perturbations. Dynamic TFs and IgSF genes were defined as ones with differential expression

517 between consecutive timepoints. Cell-type dependent genes were defined as ones with differential

518 expression in at least one pairwise comparison amongst all lamina neurons. Cell-type specific 
519 genes were defined as ones that are differentially expressed in all pairwise comparison including

520 one lamina neuron-type. Bonferroni adjustment was used for multiple testing correction.

\section{$521 \quad$ Reactome pathway analysis}

522 Dynamic transcription factors (separately for each cell-type) were entered into the

523 'Analyze Data' tool on reactome.org (https://reactome.org/PathwayBrowser/\#TOOL=AT).

524 Reactome pathway analysis for Drosophila melanogaster was then run with default parameters.

\section{TF binding site analysis}

526 i-cisTarget (https://gbiomed.kuleuven.be/apps/lcb/i-cisTarget/) ${ }^{24}$ was used (using default

527 parameters) to identify $\mathrm{TF}$ binding sites enriched within putative cis-regulatory regions and

528 ATAC-Seq peaks. dm6 version of the Drosophila genome, and version 5 of the i-cisTarget

529 database were used for all analyses. All ATAC-Seq peaks overlapping with transcription start sites

530 were excluded from TF binding site analyses to reduce the occurrence of promoter-enriched

531 sequence motifs in i-cisTarget results.

532 All EcR-pathway TF binding motifs enriched within different sets of ATAC-Seq peaks

533 were additionally verified using the Homer (v4.7) mergePeaks utility ${ }^{53}$. Briefly: first, the top 5000

534 occurrences of the motif across the genome were called using the homer scanMotifGenomeWide

535 utility. Second, Homer suite mergePeaks was then used to evaluate if the extent of overlap between

536 the top 5000 occurrences of the motif and the set of ATAC-Seq peaks is higher than expected by

537 chance. $p$-value $\leq 0.01$ was considered as significantly more overlap than expected by chance. 
Immunohistochemistry and microscopy

540 the following modifications: 1) For experiments involving staining of lamina neuron presynaptic

541 sites, brains were fixed using glyoxal (3.12\% glyoxal, $0.75 \%$ acetic acid and 20\% ethanol, $\mathrm{pH}$

542 adjusted to 5.0) for 30' at RT and then washed 3X with PBST. 2) All images were acquired using

543 an LSM880 confocal microscope.

544 Primary antibodies used in this study were: mAb24B10 (1:20, DSHB), rabbit anti-NetB

545 (1:500, gift from Akin Lab), rabbit anti-dsRed (1:400, Clontech 632496), chicken anti-GFP

546 (1:1000, Abcam ab13970), rat anti-Flag (1:200, Novus Biologicals, NBP1-06712), mouse anti-

547 EcR-B1 (1:20, DSHB AD4.4), mouse anti-EcR-A (1:10, DSHB 15G1a), rabbit anti-Hr3 (1:50, gift

548 from Thummel Lab), mouse anti-svp (1:20, DSHB 5B11), rabbit anti-erm (1:100, gift from Wang

549 Lab), rat anti-bab2 (1:500, gift from Laski Lab), mouse anti-V5 (1:200, BioRad MCA1360),

550 guinea pig anti-Bsh (1:400, see Tan et al. 2015).

$551 \quad$ Secondary antibodies used in this study were: goat anti-mouse 488 (1:500, ThermoFisher

552 A-32723), goat anti-mouse 568 (1:500, ThermoFisher A-11031), goat anti-mouse 647 (1:500,

553 ThermoFisher A-21235), goat anti-chicken 488 (1:1000, ThermoFisher A-11039), goat anti-rabbit

554568 (1:500, ThermoFisher A-11011), goat anti-guinea pig 647 (1:500, ThermoFisher A-21450),

555 goat anti-rabbit 647 (1:200, ThermoFisher A-32733), goat anti-rat 568 (1:500, ThermoFisher A556 11077).

\section{Image analysis}

All images for figures were created using ImageJ (v2.1.0). Details for quantification of 559 phenotypes are given below. 
R8 targeting

561

Imaris (v9.1.2) was used to measure the distance of R8 terminals from the top of the

562 medulla (M0), as well as the distance of $6^{\text {th }}$ medulla layer (M6) from the top of the medulla. The

563 latter was estimated using mAb24B10 staining, which labels R7 neurons that terminate in M6.

564 Values reported on the X-axis of Fig. 3c are (R8-M0)/ (M6-M0).

$565 \quad$ L5 morphological defects

566 Imaris was used to visualize individual neurons. Each neuron was then manually assigned

567 to one of the stated categories.

568 Quantification of BRP puncta in the lamina

569 The distance between the proximal end of the lamina and the distal end of the lamina was

570 measured using Imaris individually for all lamina cartridges. A line was drawn at the measured

571 distance divided by 2. All BRP puncta more distal of this line were counted manually.

\section{$572 \quad$ Ex vivo culture of dissected brains}

573 We used a protocol similar to the one described in Özel et al. ${ }^{28}$ Briefly, brains were 574 dissected from 22hAPF pupae in pre-warmed medium (ThermoFisher \#2172004) with either

575 1:1000 dilution of 1mg/ml 20-HydroxyEcdysone (dissolved in 100\% Ethanol, Sigma H5142) or 576 equivalent volume of $100 \%$ ethanol. Brains were then incubated in $\sim 200$ ul of medium \pm 20 -

577 HydroxyEcdysone in 96 well plates for $26 \mathrm{~h}$ at $25^{\circ} \mathrm{C}$ in a humidified chamber. Thereafter, brains

578 were fixed, stained and imaged using the aforementioned protocols for immunohistochemistry and 579 microscopy.

580 In the presence of 20-HydroxyEcdysone in the media, strong expression of EcR-B1 and $581 \mathrm{Hr} 3$ is observed throughout the optic lobe, while no expression of Ftz-f1 is seen (Extended data 
582 Fig. 13a). When 20-HydroxyEcdysone is omitted from the culture medium, no expression of $\mathrm{Hr} 3$

583 is seen (as expected), however weak induction of Ftz-f1 is observed. In vivo, by 22hAPF (when

584 brains are removed from pupae), Ecdysone levels have begun to rise and may be sufficient to

585 activate EcR-B1 in neurons ${ }^{21}$. As $\mathrm{Hr} 3$ activation requires a subsequent drop in Ecdysone titers ${ }^{17}$,

586 we expect the weak expression of Ftz-f1 may be due to the transfer of $22 \mathrm{hAPF}$ brains to an

587 Ecdysone-free medium.

\section{$588 \quad$ Other statistics}

To compare the dynamics of genes affected by $\mathrm{EcR}^{\mathrm{DN}}$ (fold change $\geq 2$, $\mathrm{p}$-value $\leq 0.05$

590 between WT and $E c R^{\mathrm{DN}}$ at any time point) and genes unaffected by $E c R^{\mathrm{DN}}$, normalized wildtype

591 expression for each gene was divided by the maximum expression at any time point throughout

592 development. Variance was then calculated for each gene over time. Two-tailed Student's t-test

593 was then used to calculate the statistical difference between the distributions of variance for these

594 two sets of genes. This was done separately for each cell-type.

595 Hypergeometric test was used to evaluate enrichment of targets of Erm within L3-specific

596 targets of EcR. The set of genes expressed in a L3 neurons across development was taken as the

597 universal set $(\mathrm{N})$. Then number of targets of Erm from Peng et al. ${ }^{32}(\mathrm{~m})$ and number of L3-specific

598 targets of EcR (n) were used as the number of successes in population and sample size respectively.

$599 \quad$ For all box-plots, solid line depicts median, while the upper and lower bounds of the box

600 depict the third and first quantile of the data spread respectively.

601 Kolmogorov-Smirnov (KS) test (Fig. 3c, 16c), two-tailed Student's t-test (Extended data

602 Fig. 7e, 7f, 11, 15a), two-tailed Fisher's exact test (Fig. 3e, 4a) were performed using the following 
603 basic R functions (R 3.6.1) respectively: ks.test, t.test, fisher.test. Raw data for all analyses is

604 available upon request.

\section{Data and code availability}

All raw sequencing data and codes will be provided upon request. They will be made

607 publicly available prior to publication. 
a

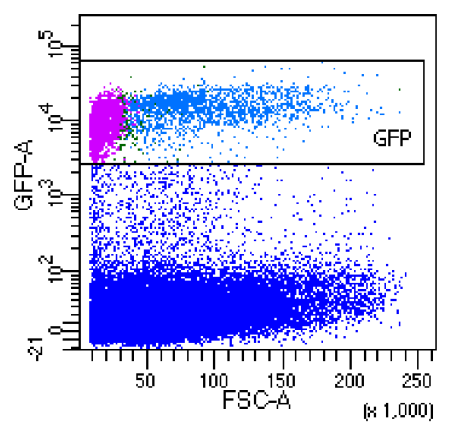

b

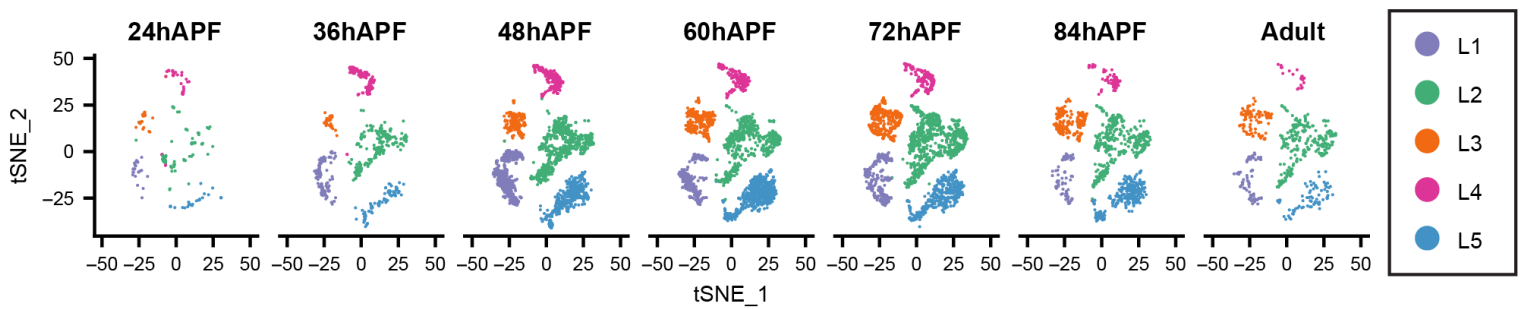

C

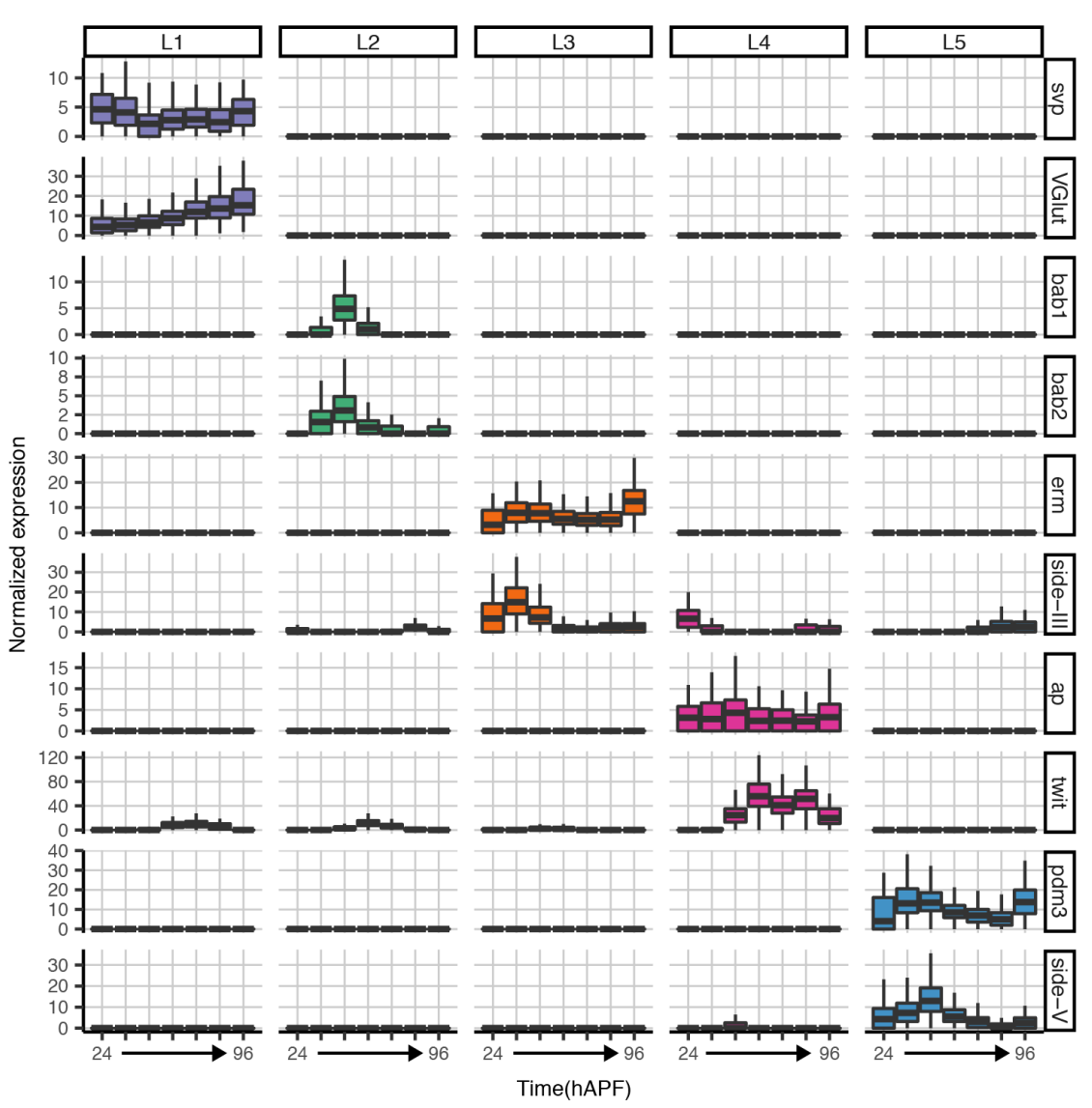


610 neurons by FACS. 'Cells' highlighted in purple were excluded despite being GFP+ due to their

611 small size. b, tSNE plots showing L1-L5 clusters at 24hAPF, 36hAPF, 48hAPF, 60hAPF, 72hAPF,

612 84hAPF and 96hAPF (Adult). c, Normalized expression of previously identified lamina neuron-

613 type-specific genes in L1-L5 clusters identified at each time point over development (see Tan et

$\left.614 a l .{ }^{12}\right)$. 
a

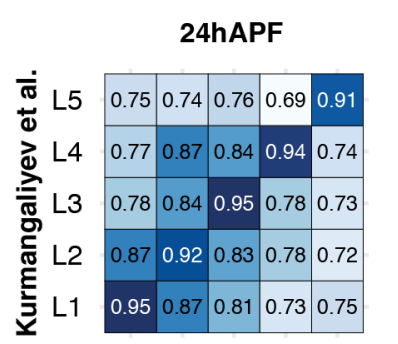

Lamina neuron sc-RNA-seq comparison

L1 L2 L3 L4 L5

This study
72hAPF

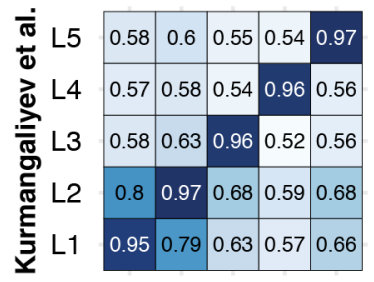

L1 L2 L3 L4 L5 This study

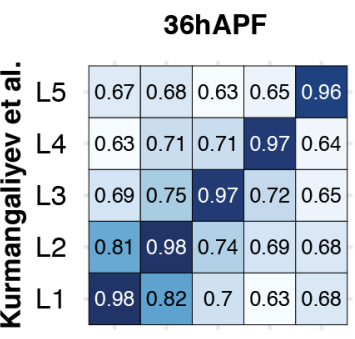

L1 L2 L3 L4 L5

This study

84hAPF

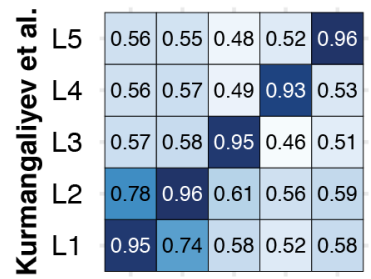

L1 L2 L3 L4 L5 This study

b Comparison of L1 bulk RNA-seq biological replicates

\begin{tabular}{l|l|l|l|l|l|l|l|}
\hline 72hAPF-2 & 0.36 & 0.36 & 0.76 & 0.8 & 0.81 & 0.98 \\
\hline
\end{tabular}

\begin{tabular}{l|l|l|l|l|l|l|l|}
\hline 72hAPF-1 & 0.33 & 0.34 & 0.74 & 0.78 & 0.79 & 1 & 0.98 \\
\hline
\end{tabular}

\begin{tabular}{l|l|l|l|l|l|l|l|} 
60hAPF-3 & 0.56 & 0.57 & 0.98 & 0.98 & 1 & 0.79 & 0.81
\end{tabular}

\begin{tabular}{l|l|l|l|l|l|l|l}
$60 h A P F-2$ & 0.57 & 0.58 & 0.98 & 1 & 0.98 & 0.78 & 0.8
\end{tabular}

\begin{tabular}{l|l|l|l|l|l|l|l}
60 hAPF-1 & 0.62 & 0.62 & 1 & 0.98 & 0.98 & 0.74 & 0.76
\end{tabular}

\begin{tabular}{l|l|l|l|l|l|l|l|} 
40hAPF-2 & 0.98 & 1 & 0.62 & 0.58 & 0.57 & 0.34 & 0.36
\end{tabular}

\begin{tabular}{l|l|l|l|l|l|l|l|} 
40hAPF-1 & 1 & 0.98 & 0.62 & 0.57 & 0.56 & 0.33 & 0.36 \\
\cline { 2 - 6 }
\end{tabular}

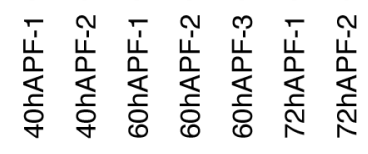

Spearman Correlation

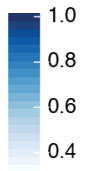

C

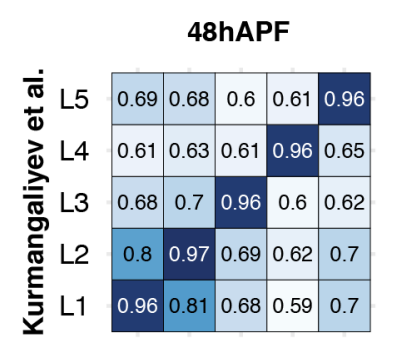

L1 L2 L3 L4 L5

This study

96hAPF

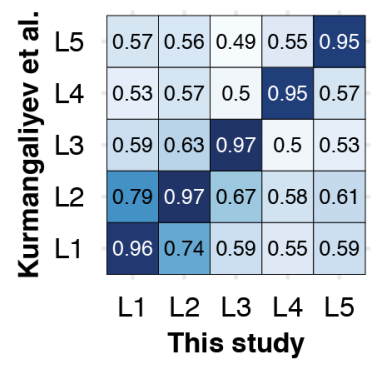

60hAPF

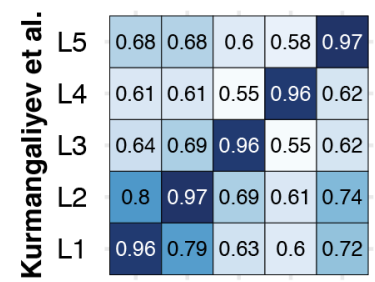

L1 L2 L3 L4 L5

This study

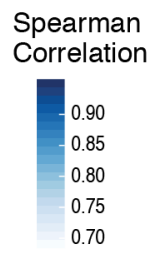

Comparison of L1 scRNA-seq and bulk RNA-seq

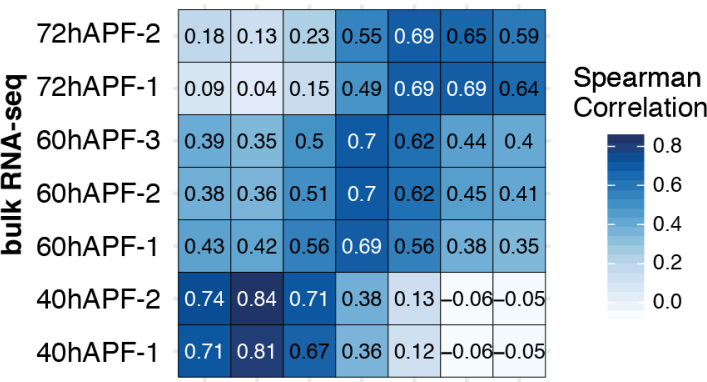

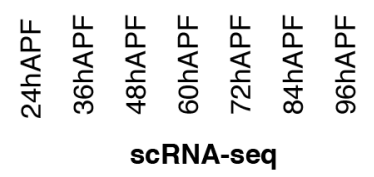

615 Extended data Fig. 2 | Comparison of scRNA-Seq-derived transcriptome with other datasets.

616 a, Comparison of lamina neuron transcriptomes generated by scRNA-Seq in this study and by

617 scRNA-Seq in Kurmangaliyev et al. $\mathbf{b}$, Comparison of replicates of bulk-RNA-Seq of L1 neurons

618 at 40hAPF, 60hAPF and 72hAPF (this study). c, Comparison of lamina neuron transcriptomes

619 generated by scRNA-Seq in this study and by bulk RNA-Seq, also this study. See Methods for

620 calculation of Spearman correlation. 

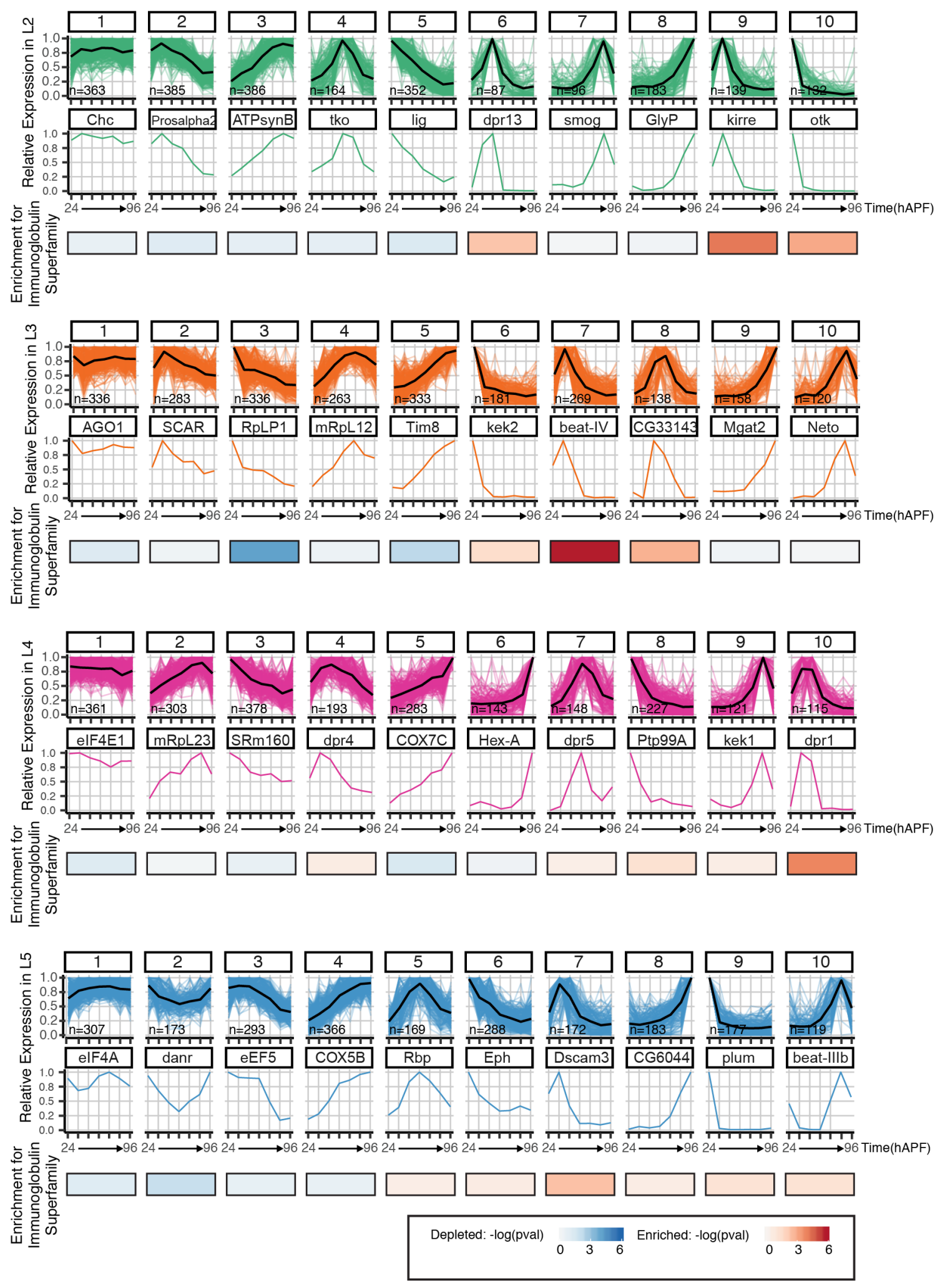

621 Extended data Fig. 3 | Gene groups generated via k-means clustering. Gene groups generated

622 via k-means clustering using expression dynamics in L2-L5 neurons (see Methods). Relative 
623 expression over development for each gene in the group is shown as a line plot (colored lines),

624 with the mean shown as a black line. n, number of genes in the group. A gene from each group is

625 shown. Heat map depicts enrichment (- $\log _{10}$ p-values) of Immunoglobulin Superfamily genes over

626 that expected by chance (see Methods). 
bioRxiv preprint doi: https://doi.org/10.1101/2020.09.18.304410; this version posted September 19, 2020. The copyright holder for this preprint (which was not certified by peer review) is the author/funder, who has granted bioRxiv a license to display the preprint in perpetuity. It is made available under aCC-BY-NC-ND 4.0 International license.

a

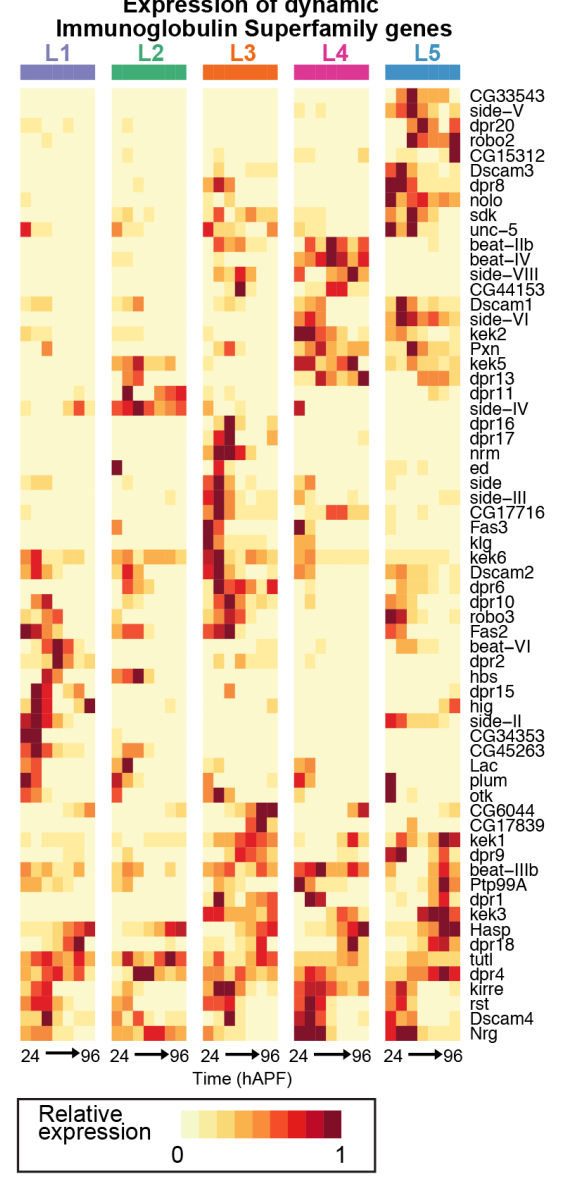

Expression of dynamic transcription factors

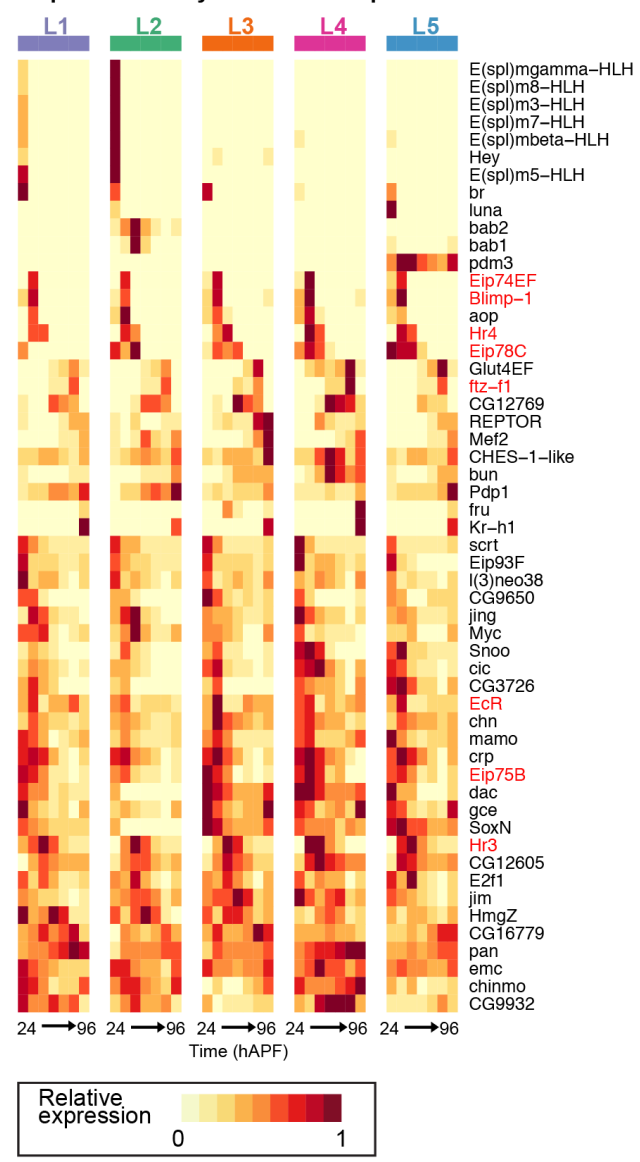

C

\begin{tabular}{|l|l|}
\hline Reactome Pathway in L1 & $\mathbf{p}$-value \\
\hline Nuclear Receptor transcription pathway & $7.5 \times 10^{-6}$ \\
\hline SUMOylation of intracellular receptors & $1.1 \times 10^{-4}$ \\
\hline Reactome Pathway in L2 & $\mathbf{p}$-value \\
\hline Nuclear Receptor transcription pathway & $7.4 \times 10^{-8}$ \\
\hline SUMOylation of intracellular receptors & $1.7 \times 10^{-7}$ \\
\hline Reactome Pathway in L3 & $\mathbf{p}$-value \\
\hline Nuclear Receptor transcription pathway & $1.3 \times 10^{-7}$ \\
\hline SUMOylation of intracellular receptors & $7.4 \times 10^{-7}$ \\
\hline SUMO E3 ligases SUMOylate target proteins & $1.2 \times 10^{-3}$ \\
\hline SUMOylation & $1.3 \times 10^{-3}$ \\
\hline Generic Transcription Pathway & $3.6 \times 10^{-3}$ \\
\hline Reactome Pathway in L4 & $\mathbf{p}$-value \\
\hline Nuclear Receptor transcription pathway & $1.3 \times 10^{-9}$ \\
\hline SUMOylation of intracellular receptors & $6.0 \times 10^{-9}$ \\
\hline SUMO E3 ligases SUMOylate target proteins & $6.4 \times 10^{-5}$ \\
\hline SUMOylation & $7.7 \times 10^{-5}$ \\
\hline Generic Transcription Pathway & $4.4 \times 10^{-4}$ \\
\hline RNA polymerase II transcription & $2.1 \times 10^{-3}$ \\
\hline Gene expression (transcription) & $5.3 \times 10^{-3}$ \\
\hline
\end{tabular}


629 Superfamily proteins with dynamic expression in at least one lamina neuron-type over

630 development (64 genes, $\geq 2$-fold change in expression between any consecutive time points, $\mathrm{p}$ -

631 value $<0.05)$. Each row represents a different gene. Each column is a different time-point every

$63212 \mathrm{~h}$ from 24 to 96 hAPF (Adult). Note the cell-type dependent patterns of expression of IgSF

633 coding genes. b, Heat map showing relative expression of dynamic transcription factors (see

634 Methods). Many members of the Ecdysone signaling pathway are highlighted in red. c, Reactome

635 pathway analysis of dynamic TFs showing all enriched categories ( $p$-value $<0.01)$. Enrichment of

636 the Nuclear Receptor Transcription Pathway is highlighted in L1-L5 neurons. 

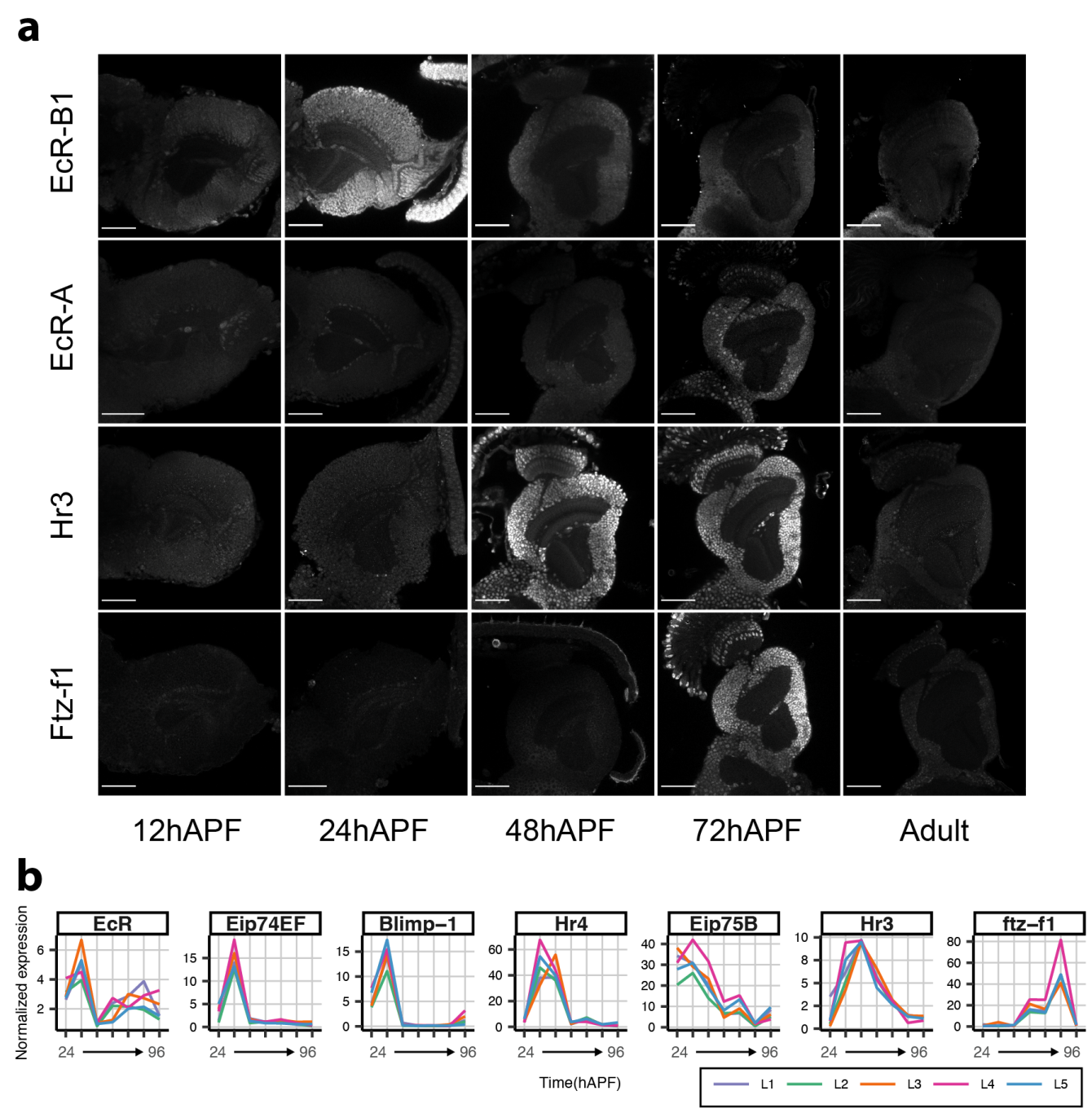

C

EcR-B1 specific exon

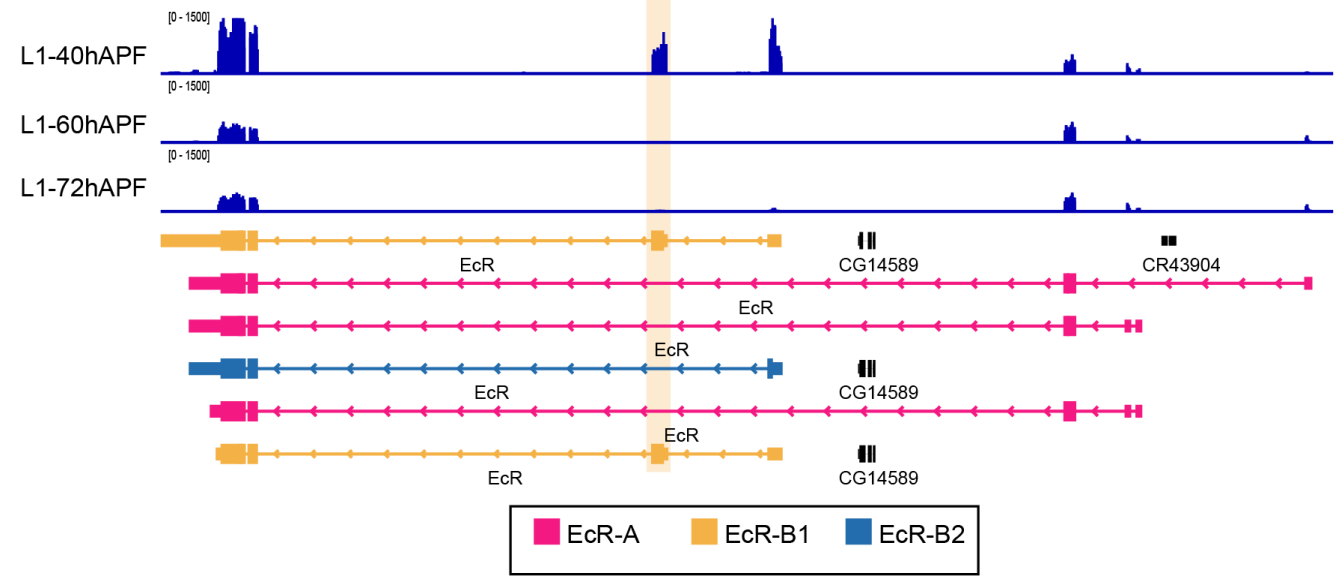

637 Extended data Fig. 5 | Expression of TFs in the Ecdysone cascade. a, Images showing immuno-

638 staining for EcR-B1, EcR-A, Hr3 and Ftz-f1 at 12hAPF, 24hAPF, 48hAPF, 72hAPF and in adults. 
639 Note that an anti-GFP antibody was used to stain for Ftz-f1 in fly lines carrying a BAC with GFP-

640 tagged Ftz-f1 and endogenous cis-regulatory sites (see Supplementary Table 1). Scale bar, $50 \mu \mathrm{m}$.

641 b, Line plots of expression of TFs. c, Read pile-up in the EcR locus showing change of isoform

642 from predominantly EcR-B1 to EcR-A between 40hAPF and 60hAPF. 

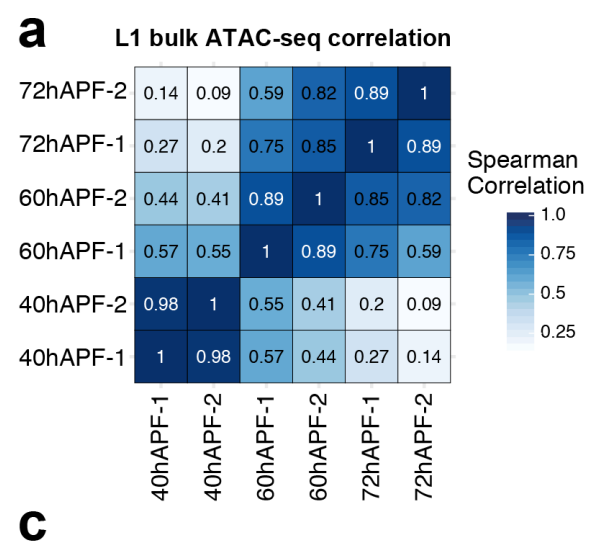

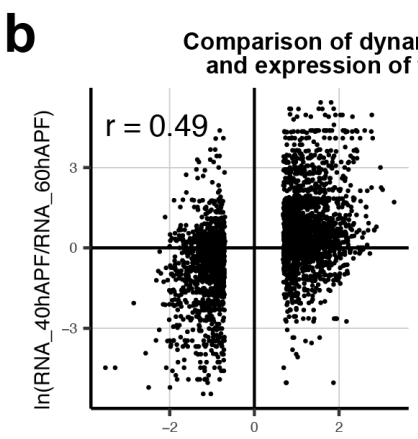

In(ATAC_40hAPF/ATAC_60hAPF)

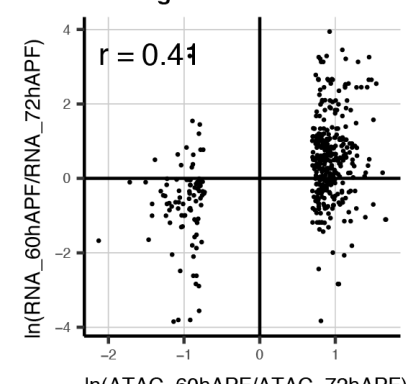

In(ATAC_60hAPF/ATAC_72hAPF)
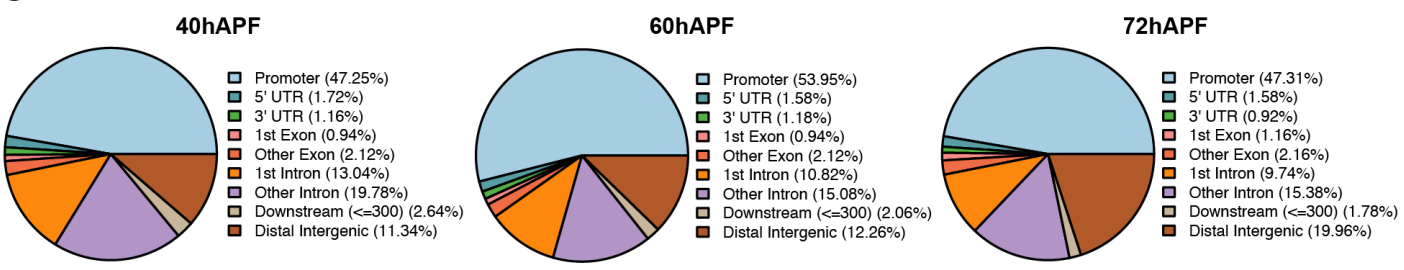

d
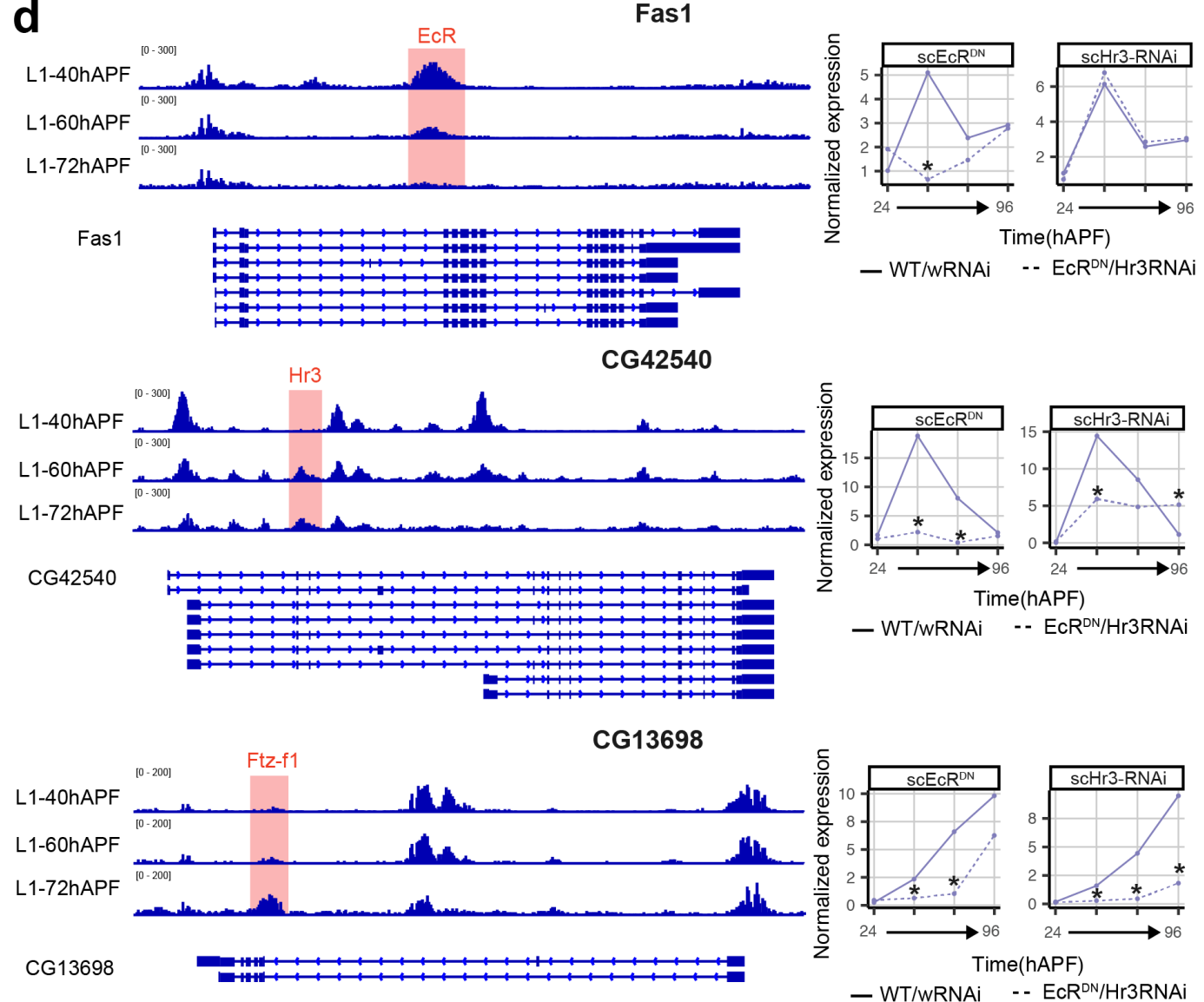

Extended data Fig. 6 | ATAC-Seq analysis of developing L1 neurons. a, Comparison of

644 replicates of bulk ATAC-Seq data of L1 neurons at 40hAPF, 60hAPF and 72hAPF. Values shown 
645 are Spearman correlation values (see Methods). b, Comparison of change of ATAC-seq peak

646 coverage and change in expression of nearest gene. $\ln ($ fold change RPKM of nearest gene) vs

$647 \ln$ (fold change ATAC-seq peak coverage) between 40hAPF and 60hAPF, and 60hAPF and

648 72hAPF. r, Pearson's correlation coefficient. c, Distribution of the top 5000 peaks at each time

649 point between various genomic landmarks. d, Normalized ATAC-Seq read pile-ups are shown for

650 L1 neurons at 40hAPF, 60hAPF and 72hAPF for three gene loci - Fas1, CG42450, CG13698.

651 ATAC-Seq peaks with predicted binding sites for EcR, Hr3 and Ftz-f1 are highlighted. Shown

652 along with is normalized expression of these genes in control (solid line) vs EcR ${ }^{\mathrm{DN}}$ or Hr3 RNAi

653 expressing L1 neurons (dashed lines). 
a

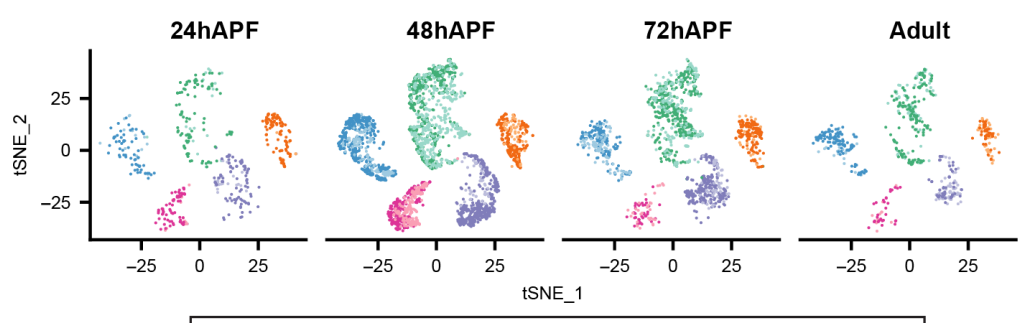

\begin{tabular}{|c|c|c|c|c|}
\hline L1 WT & L2WT & L3 WT & L4 WT & L5 WT \\
\hline L1 EcR & $\mathrm{L} 2 \mathrm{Ec} \mathrm{R}^{\mathrm{DN}}$ & L3 EcR ${ }^{D N}$ & L4 EcR ${ }^{D N}$ & $\mathrm{~L} 5 \mathrm{EcR}^{\mathrm{DN}}$ \\
\hline
\end{tabular}

b

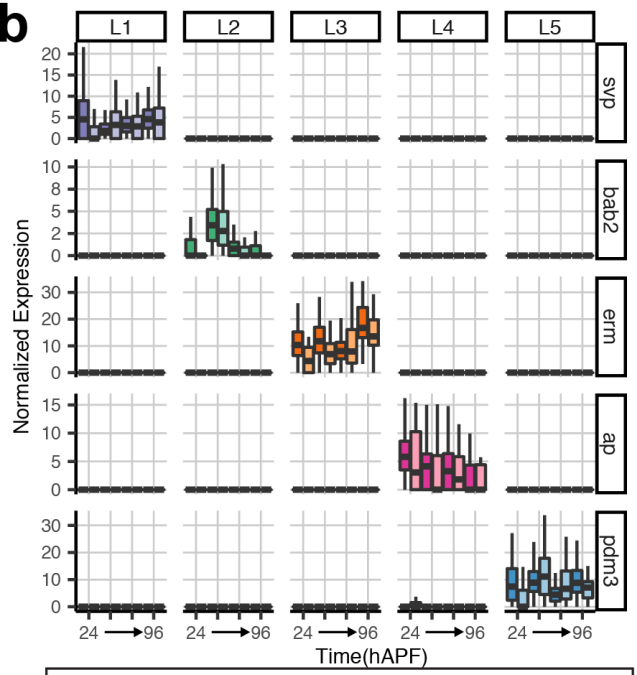

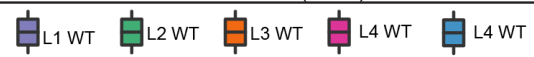

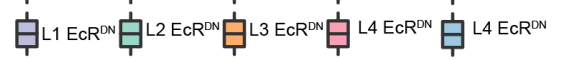

d

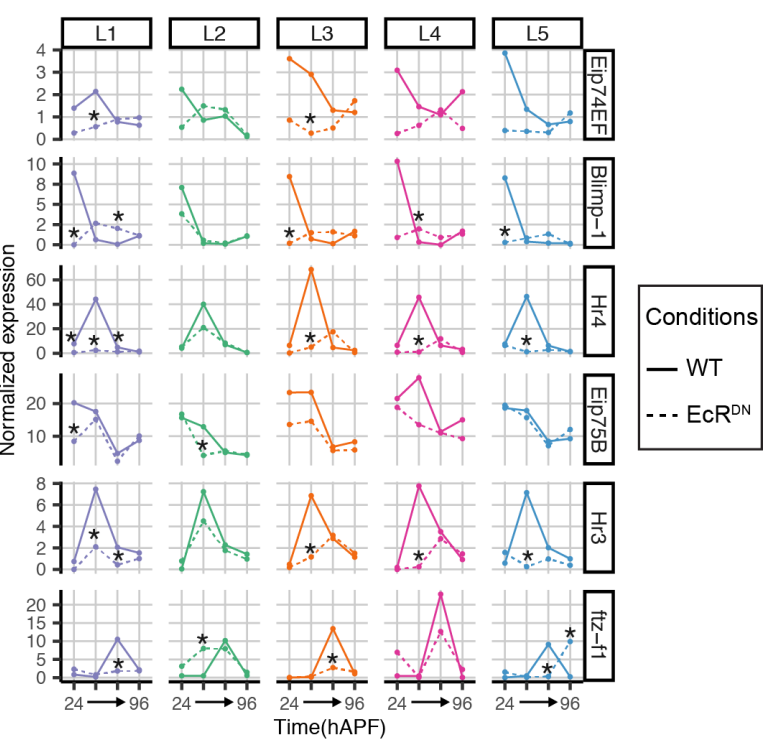

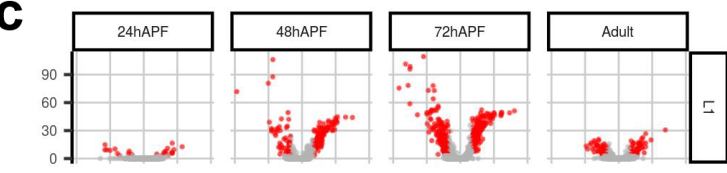
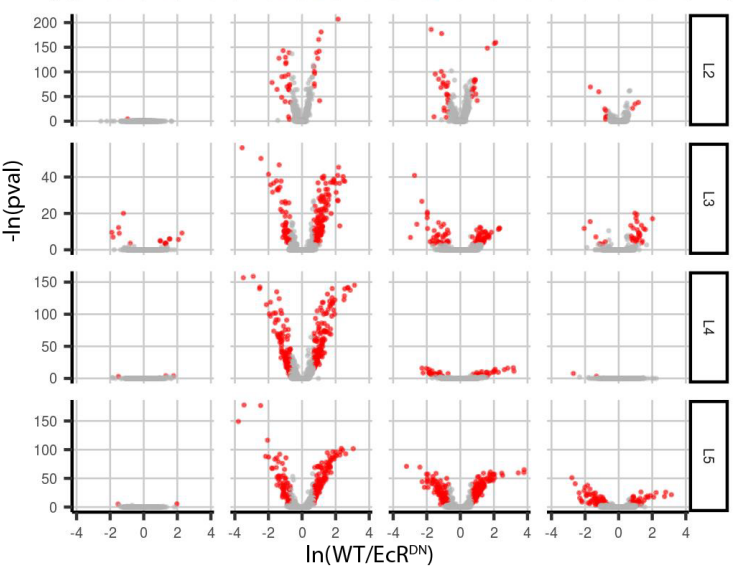

e

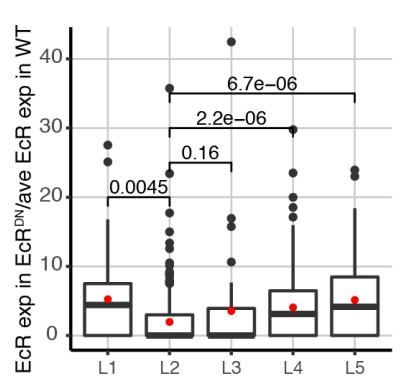

f

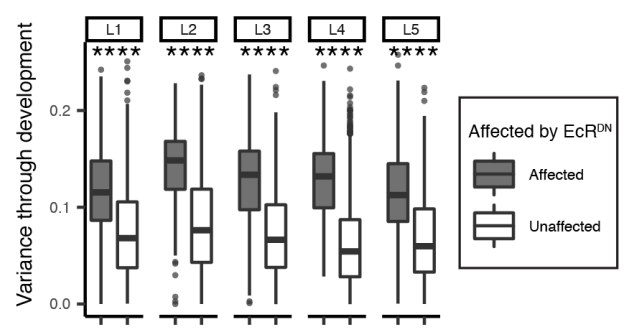


656 72hAPF, and 96hAPF (Adult). b, Expression of lamina neuron-type-specific TFs \pm EcR $^{\mathrm{DN}}$

657 expression. c, Volcano plots showing significant gene expression changes in L1-L5 neurons

658 throughout development. Red dots: fold change $\geq 2$ and $p$-value $\leq 0.05$. d, Normalized expression

659 of TFs in the Ecdysone-signaling pathway in WT (solid lines) and EcR ${ }^{\mathrm{DN}}$-expressing (dashed

660 lines) L1-L5 neurons. *, p-value $\leq 0.05$, fold change $\geq 2$. e. Expression of EcR in EcR ${ }^{\mathrm{DN}}$-expressing

661 lamina neurons at $48 \mathrm{hAPF}$ normalized to mean expression of EcR in wildtype cells at $48 \mathrm{hAPF}$

662 (done separately for each lamina neuron-type). Red dots, mean of data spread. Increase in EcR

663 expression in $\mathrm{EcR}^{\mathrm{DN}}$-expressing cells over wildtype is expected to be due to the expression of the

$664 \mathrm{EcR}^{\mathrm{DN}}$ transgene. Note the poor induction of $\mathrm{EcR}^{\mathrm{DN}}$ in $\mathrm{L} 2$ neurons. p-value from student's t-test

665 are stated in the figure for comparison between L2 and other lamina neuron-types. The difference

666 between $\mathrm{EcR}^{\mathrm{DN}}$ expression in $\mathrm{L} 2$ and L3 neurons is not significant likely due to the low cell

667 numbers of $\mathrm{EcR}^{\mathrm{DN}}$-expressing L3 neurons. f, Comparison of variance of wildtype expression of

668 genes affected or unaffected by $\mathrm{EcR}^{\mathrm{DN}}$ expression (see methods). ****, p-value $<0.0001$, student's

669 t-test. 


\section{Expression of $E c R^{D N}$ affected genes in wildtype lamina neurons}

24hAPF

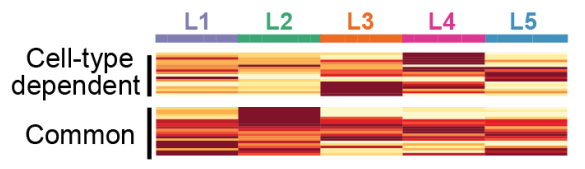

72hAPF
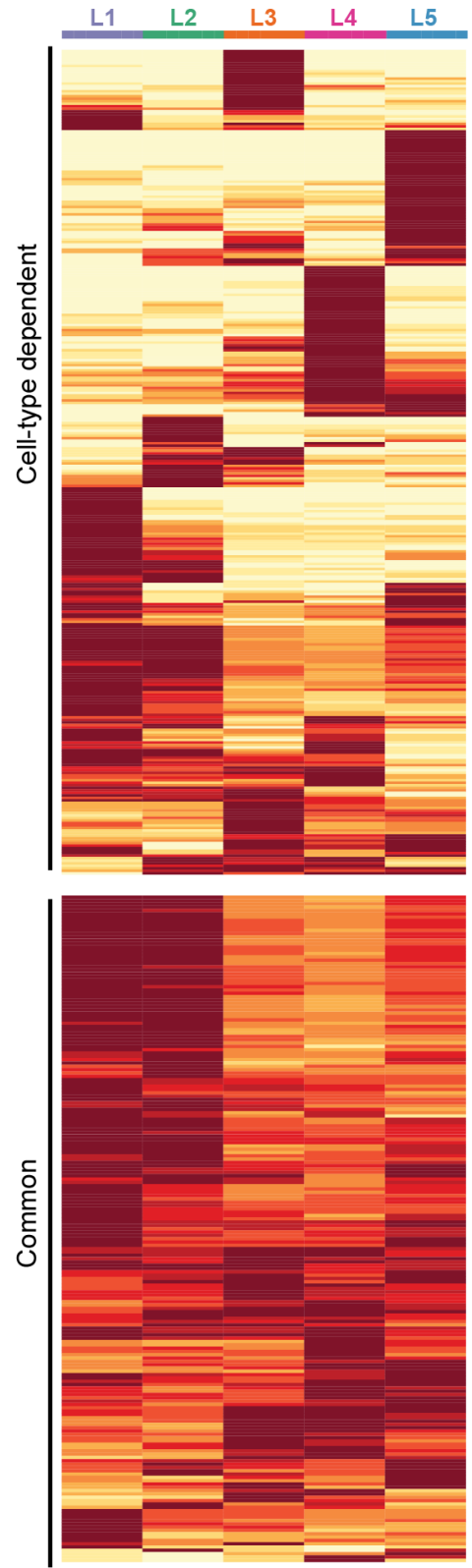

Adult
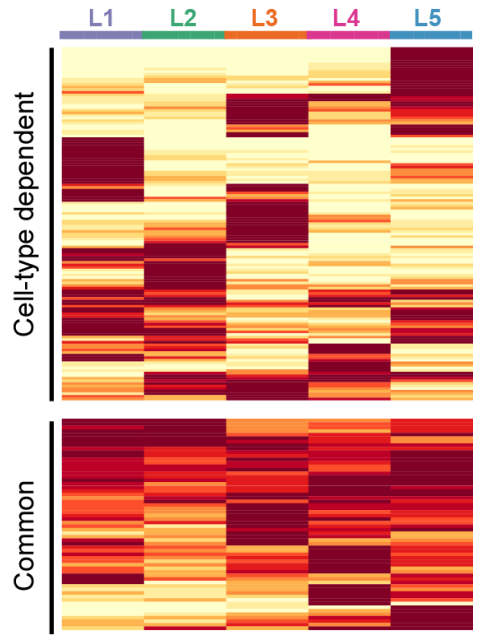

Relative

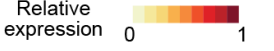

670 Extended data Fig. 8 | Cell-type dependent targets of EcR. Heat map showing relative

671 expression in wildtype cells of genes affected by $\mathrm{EcR}^{\mathrm{DN}}$ at $24 \mathrm{hAPF}, 72 \mathrm{hAPF}$ and in adults. Targets

672 of EcR are divided into two categories: Cell-type dependent (in any pairwise comparison fold

673 change $\geq 2$, p-value $\leq 0.05$ ) and Common targets (no significant expression difference in any

674 pairwise comparison) (see Fig. 2c and Methods). 
a
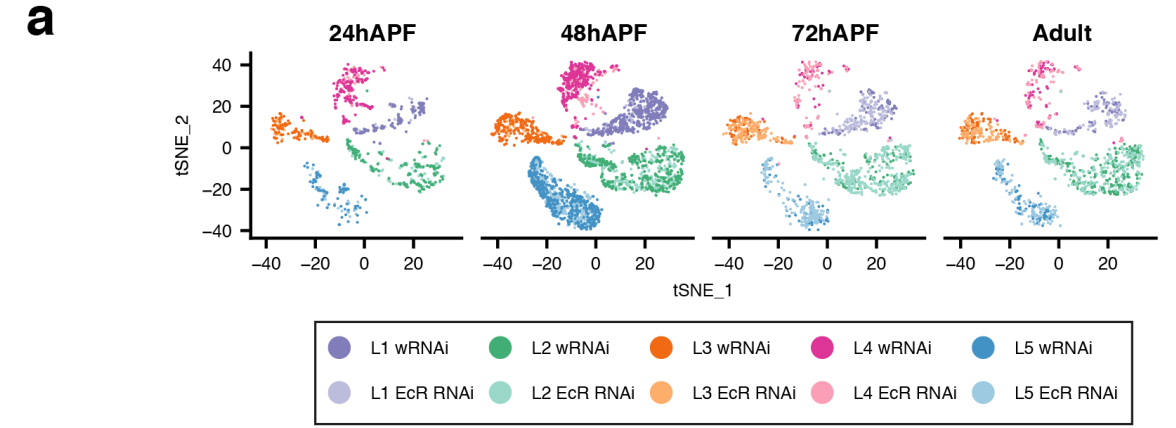

b
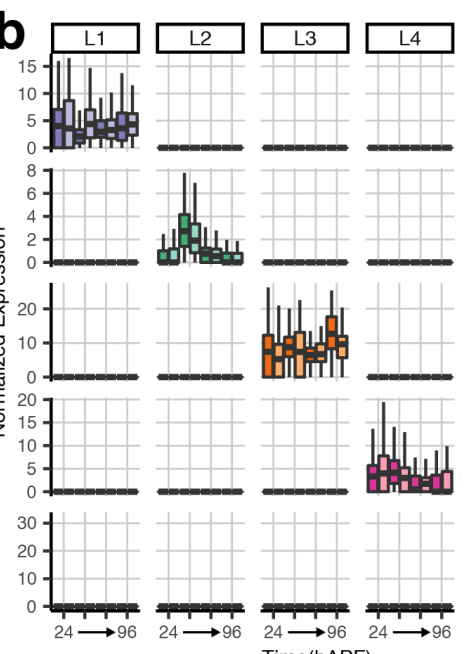

$24 \longrightarrow 96$
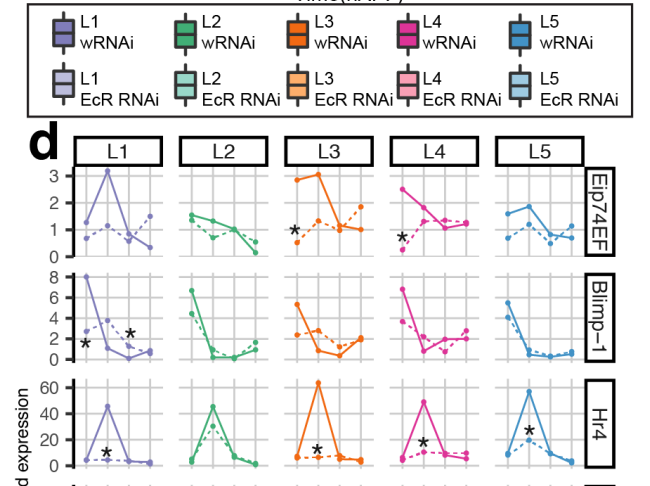

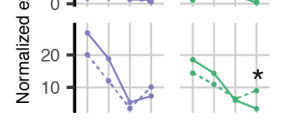
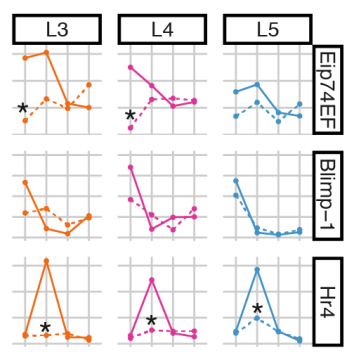

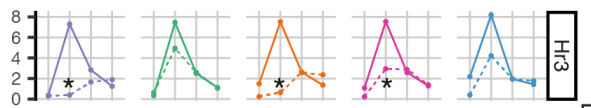

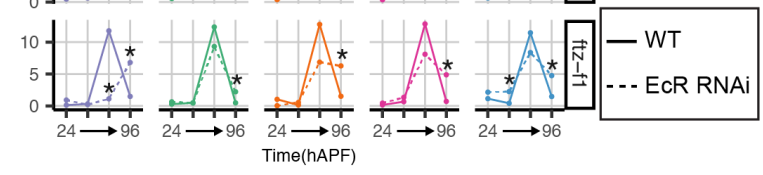

e
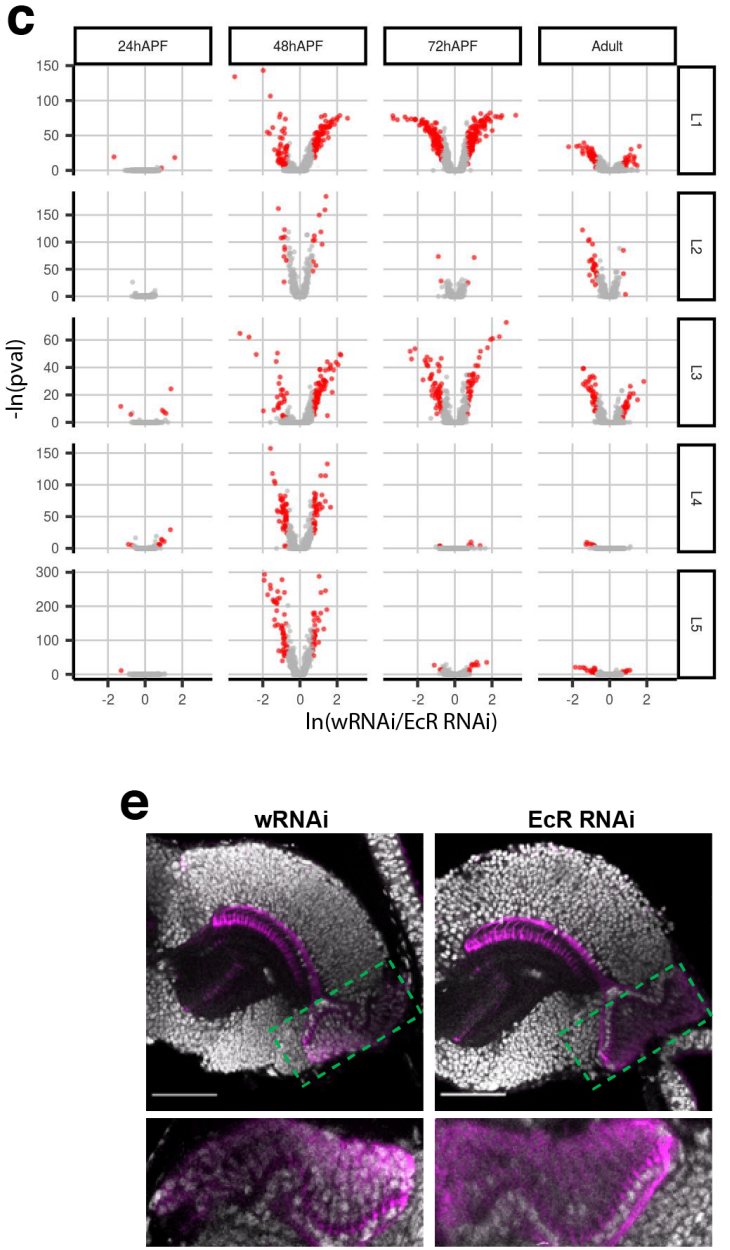

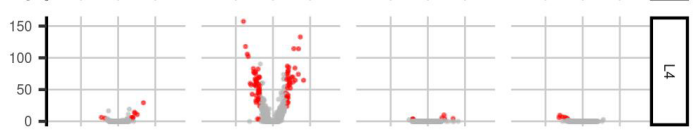

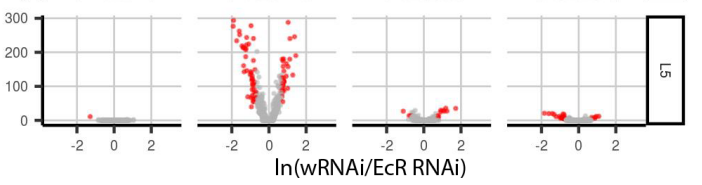

.
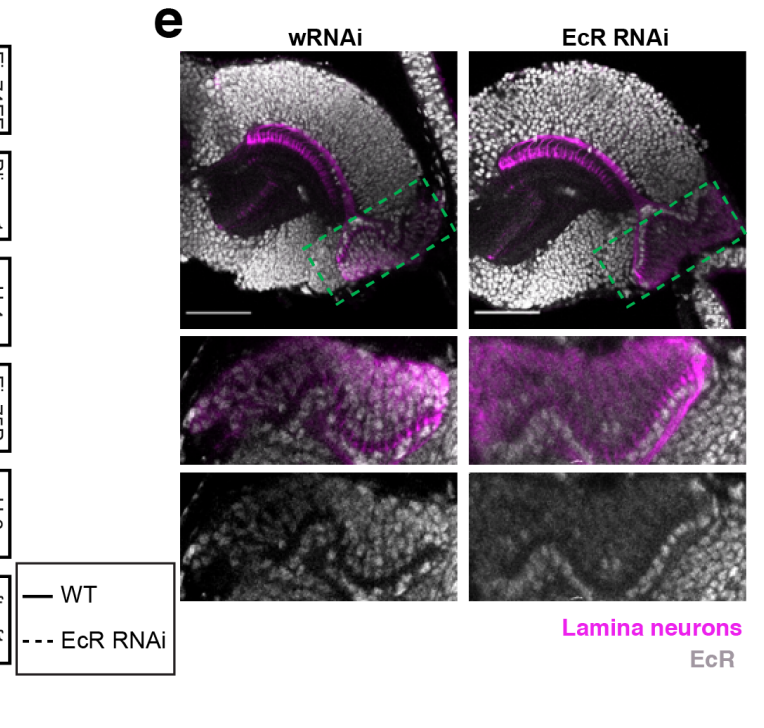

Lamina neurons

$\mathrm{EcR}$ 
677 clusters at 24hAPF, 48hAPF, 72hAPF, and 96hAPF (Adult). b, Expression of lamina neuron-type-

678 specific TFs \pm EcR RNAi expression. c, Volcano plots showing significant gene expression

679 changes in L1-L5 neurons throughout development. Red dots: fold change $\geq 2$ and p-value $\leq 0.05$.

$680 \mathrm{~d}$, Average expression of TFs in the Ecdysone-signaling pathway in wRNAi (solid lines) and EcR

681 RNAi-expressing (dashed lines) L1-L5 neurons. *, p-value $\leq 0.05$, fold change $\geq 2$. e, Image

682 showing optic lobe (top) stained using an antibody targeting all EcR isoforms (grey) at $24 \mathrm{hAPF}$.

683 Box with green dotted outline marks the region containing lamina neuron cell-bodies. This region

684 is magnified in bottom two panels. Lamina neurons are labeled in magenta. Scale bar, $50 \mu \mathrm{m}$. 
bioRxiv preprint doi: https://doi.org/10.1101/2020.09.18.304410; this version posted September 19, 2020. The copyright holder for this preprint (which was not certified by peer review) is the author/funder, who has granted bioRxiv a license to display the preprint in perpetuity. It is made available under aCC-BY-NC-ND 4.0 International license.

a

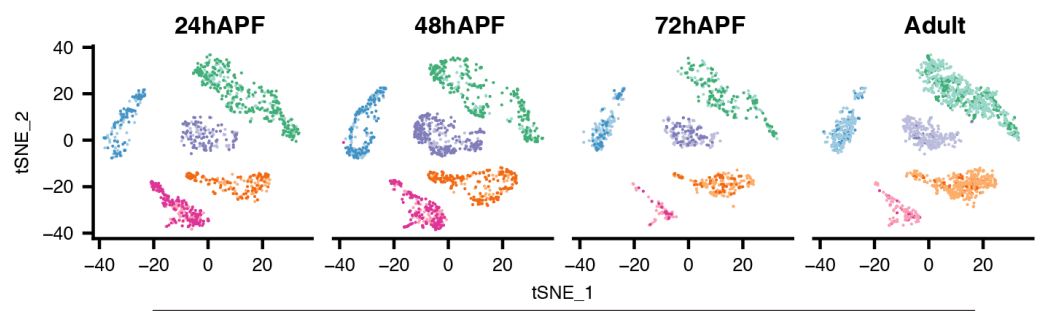

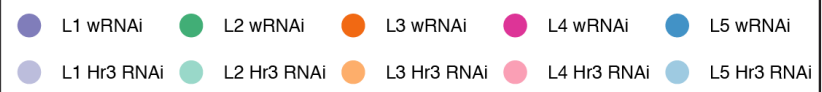

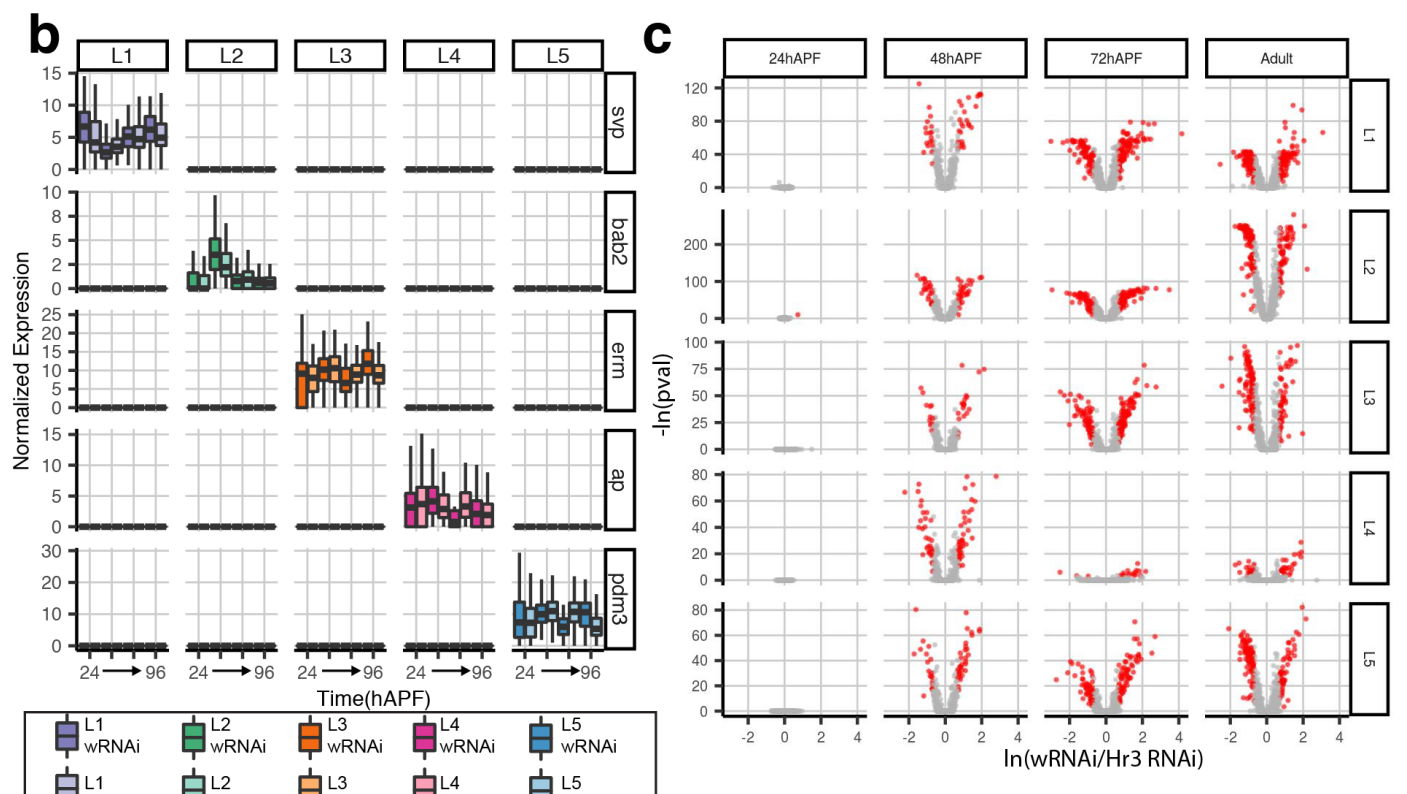

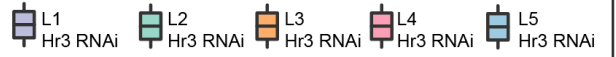
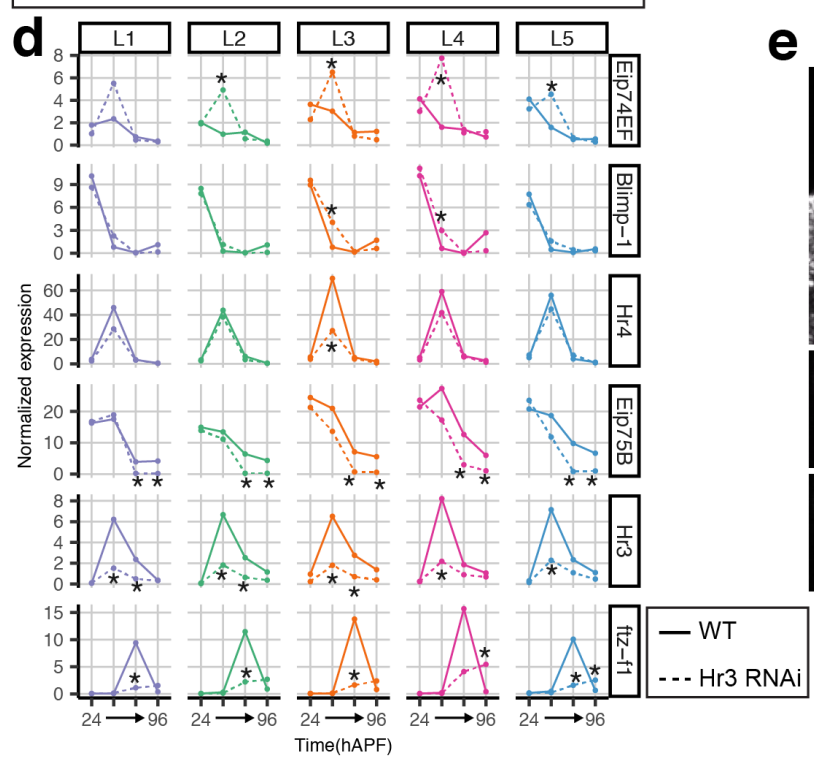

e
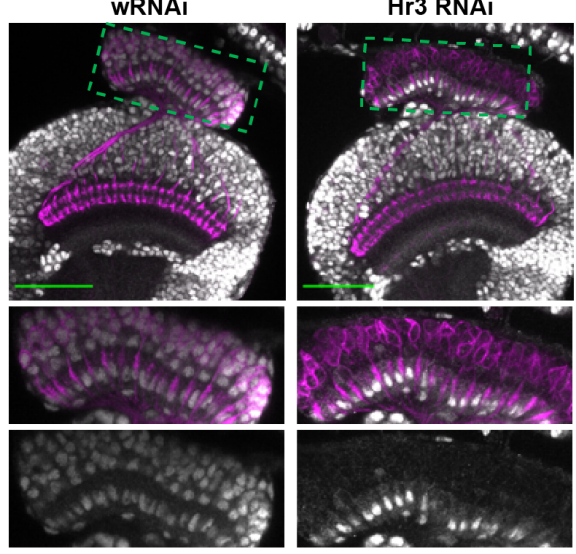

Lamina neurons

EcR 
687 clusters at 24hAPF, 48hAPF, 72hAPF, and 96hAPF (Adult). b, Expression of lamina neuron-type-

688 specific TFs \pm Hr3 RNAi expression. c, Volcano plots showing significant gene expression

689 changes in L1-L5 neurons throughout development. Red dots: fold change $\geq 2$ and p-value $\leq 0.05$.

690 d, Average expression of TFs in the Ecdysone-signaling pathway in wRNAi (solid lines) and $\mathrm{Hr} 3$

691 RNAi-expressing (dashed lines) L1-L5 neurons. *, p-value $\leq 0.05$, fold change $\geq 2$. e, Image

692 showing optic lobe (top) stained using an antibody targeting Hr3 (grey) at 48hAPF. Box with green

693 dotted outline marks the region containing lamina neuron cell-bodies. This region is magnified in

694 bottom two panels. Lamina neurons are labeled in magenta. Scale bar, $50 \mu \mathrm{m}$. Also see panel d for 695 efficiency of Hr3 knockdown. 

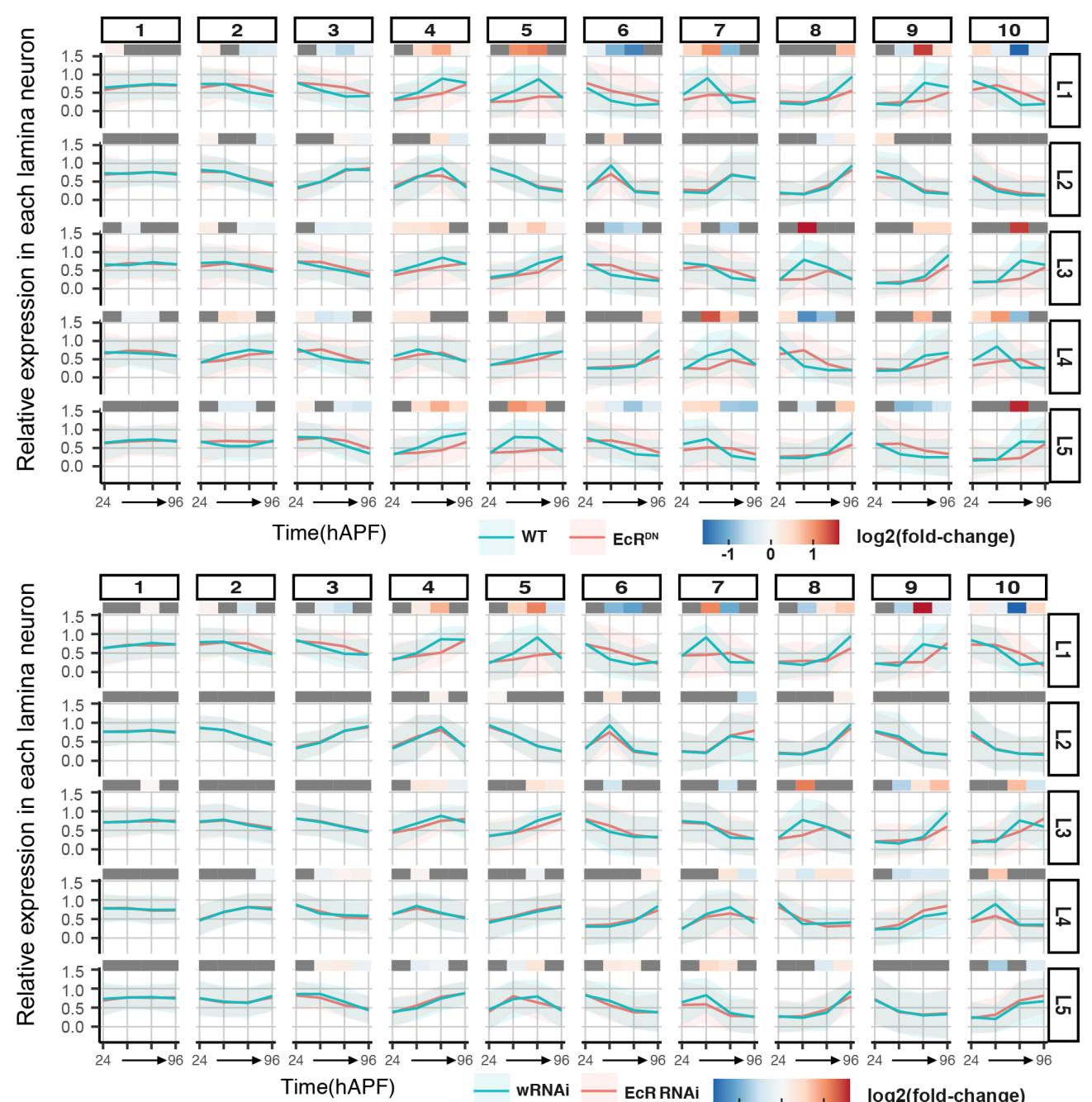

Time(hAPF)

— wRNAi - EcRRNAi ${ }_{-1}$ i $\quad 1 \quad \log 2$ (fold-change)

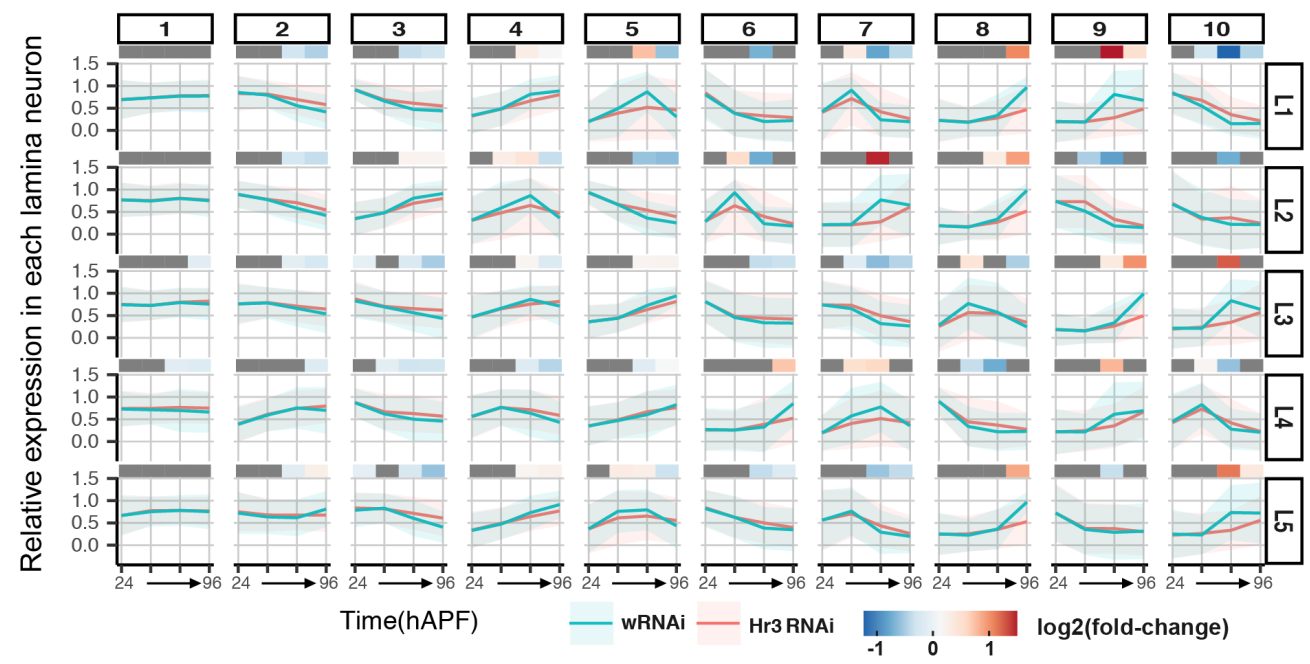


698 Hr3 activity (orange) for each gene group in L1-L5 neurons generated via k-means clustering (see

699 Fig. 1c, Extended data Fig. 3). Average behavior of genes in a group for control and perturbation

700 of EcR or Hr3 activity are shown in bold lines, while standard deviation is shown in shaded region

701 of the same color. Heat map shows $\log _{2}$ (Fold change) between average of WT and average of

702 perturbation for all comparisons where student's t-test $\mathrm{p}$-value $<0.01$. 
a

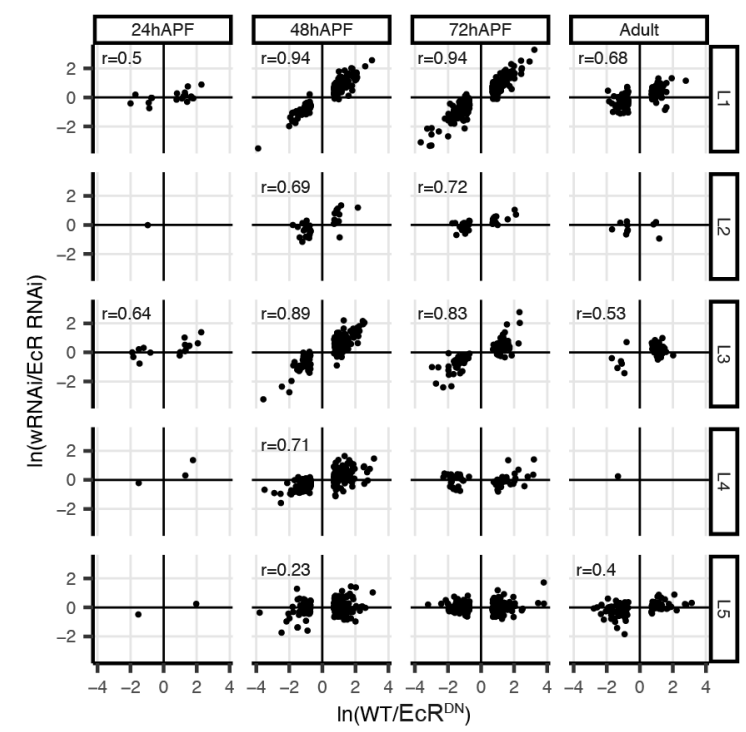

b
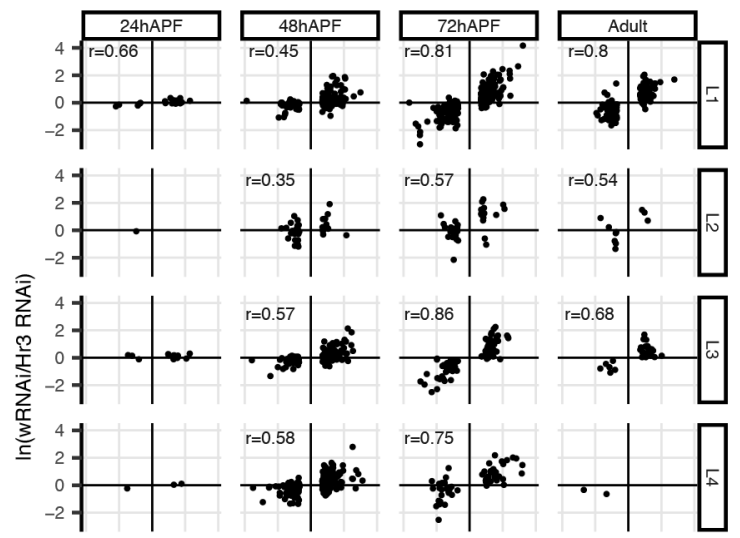

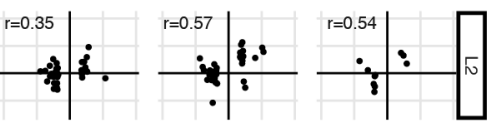
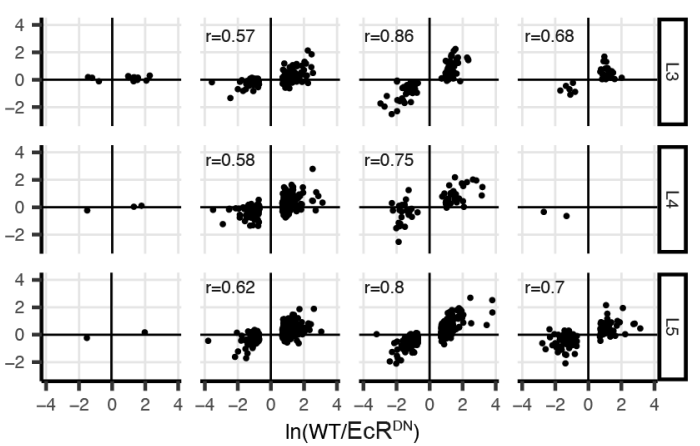

704 RNAi. a, $\ln$ (normalized expression in wRNAi/EcR RNAi) vs $\ln$ (normalized expression in

$705 \mathrm{WT} / \mathrm{EcR}^{\mathrm{DN}}$ ) for L1-L5 neurons throughout development. b, $\ln$ (normalized expression in

706 wRNAi/Hr3 RNAi) vs $\ln$ (normalized expression in $\mathrm{WT} / \mathrm{EcR}^{\mathrm{DN}}$ ) for L1-L5 neurons throughout

707 development. a, b, r, Pearson's correlation coefficient. 
bioRxiv preprint doi: https://doi.org/10.1101/2020.09.18.304410; this version posted September 19, 2020. The copyright holder for this preprint (which was not certified by peer review) is the author/funder, who has granted bioRxiv a license to display the preprint in perpetuity. It is made available under aCC-BY-NC-ND 4.0 International license.
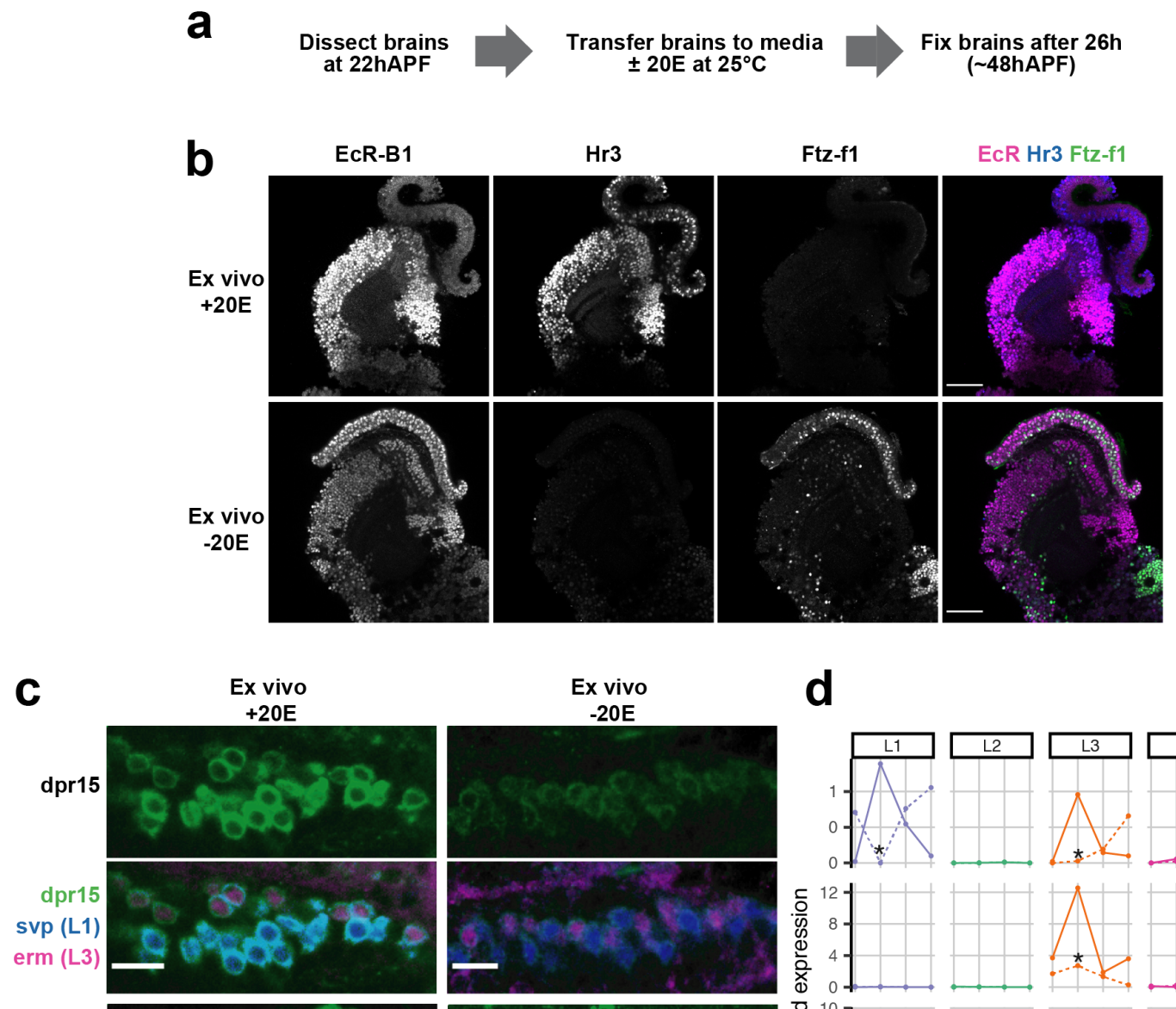

d
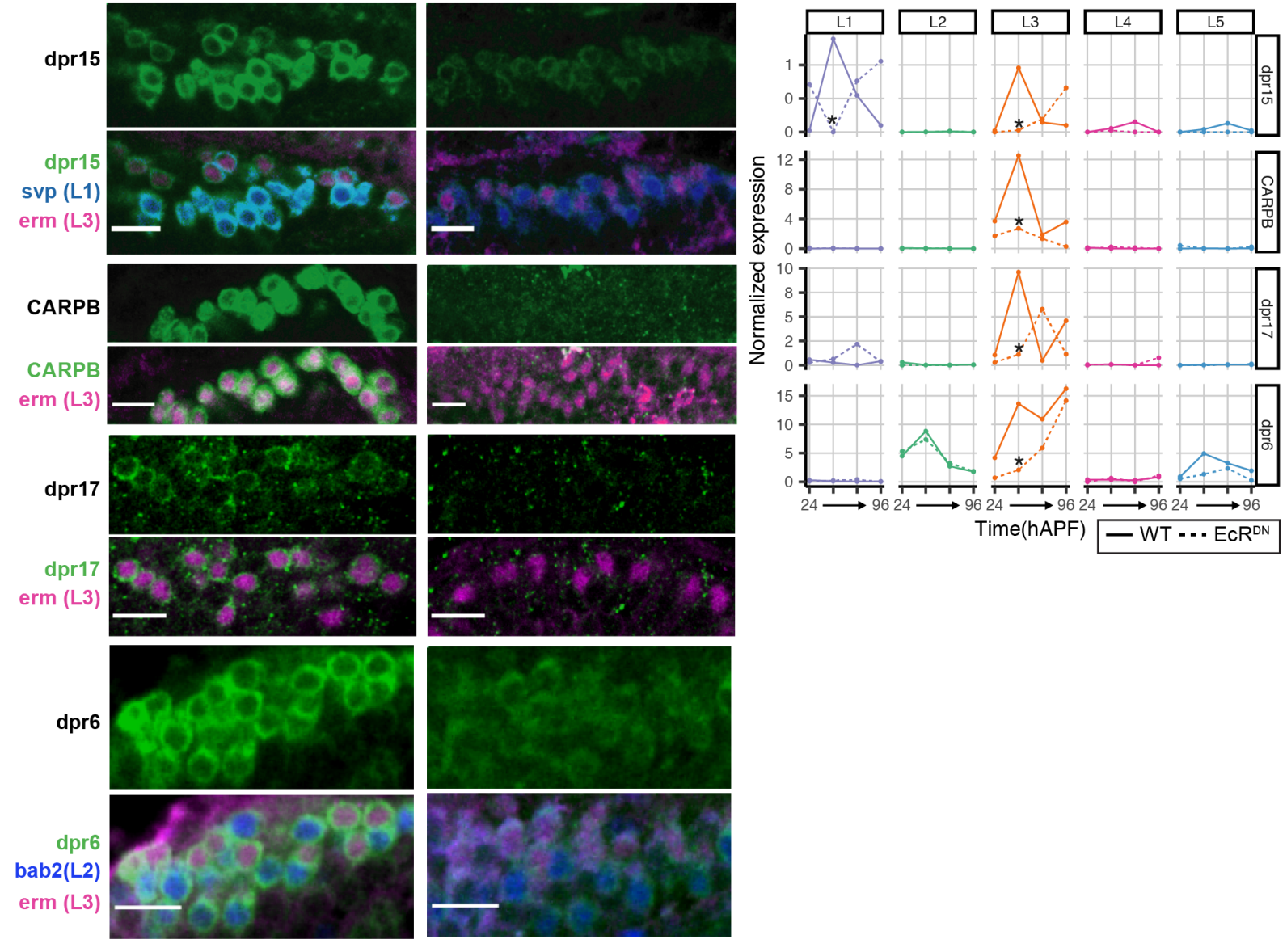

Extended data Fig. 13 | Gene expression in ex vivo brain cultures with and without Ecdysone. 
710 EcR-B1, Hr3 and Ftz-f1 in brain explants \pm inclusion of $20 \mathrm{E}$ in the culture medium. Scale bar,

$71150 \mu \mathrm{m}$. See Methods. c, Staining for protein-traps or GFP-Mimic reporter lines for dpr15, CARPB,

712 dpr17 and dpr6 (genes with cell-type-dependent patterns of expression and predicted to require

713 Ecdysone for expression from our sequencing data) \pm inclusion of $20 \mathrm{E}$ in the medium. Scale bar,

$71410 \mu \mathrm{m}$. Different lamina neurons are labeled using antibodies against lamina neuron-type-specific

715 TFs (see Extended data Fig. 1c). d, Normalized expression of dpr15, CARPB, dpr17 and dpr6 in

716 wildtype (solid lines) and $\mathrm{EcR}^{\mathrm{DN}}$-expressing (dashed lines) L1-L5 neurons throughout

717 development. *, p-value $\leq 0.05$, fold change $\geq 2$. Note that dpr6 shows Ecdysone-dependent

718 expression in L2 neurons in ex vivo cultured brains, but seems to be unaffected by $\mathrm{EcR}^{\mathrm{DN}}$

719 expression based on our sequencing data. This is consistent with low induction of $\mathrm{EcR}^{\mathrm{DN}}$ in $\mathrm{L} 2$

720 neurons using the pan-lamina Gal4 driver (see Extended data Fig. 7e). 

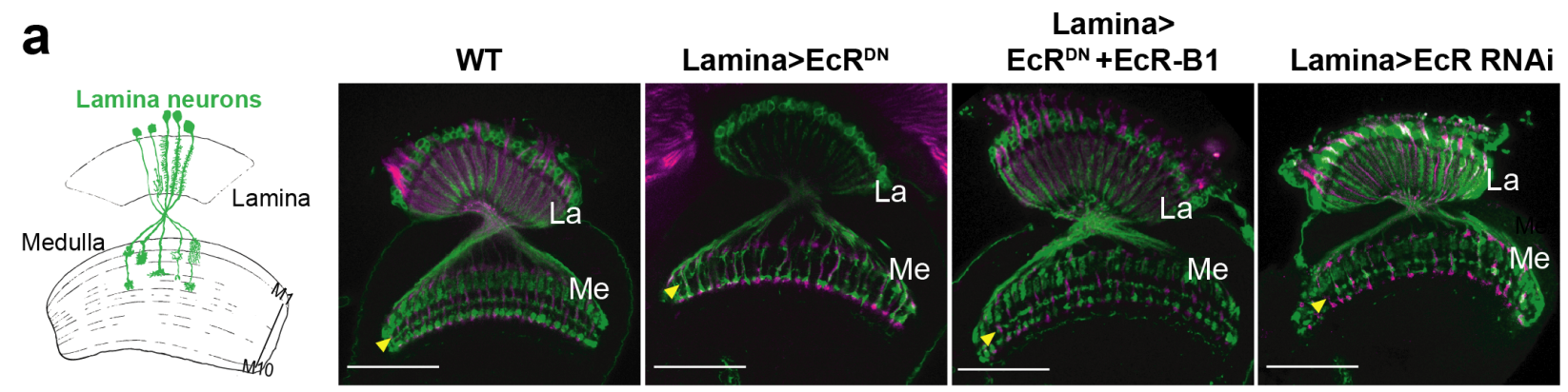

Lamina>GFP 24B10
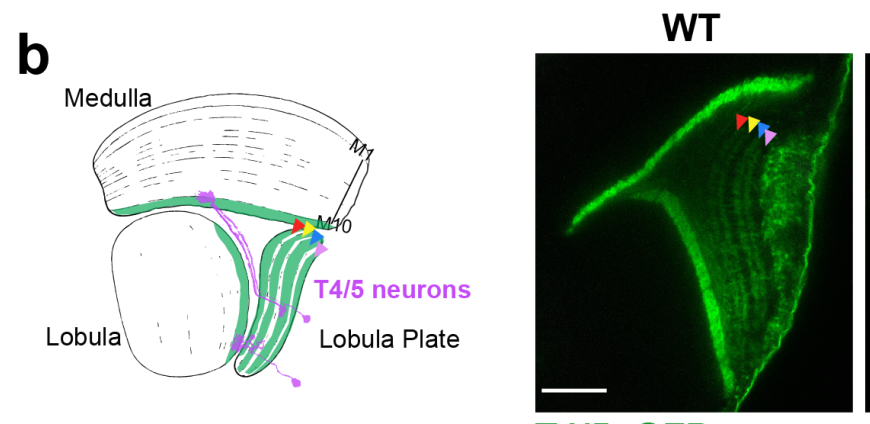

$T 4 / 5>E c R^{D N}$

T4/5>GFP

721 Extended data Fig. 14 | Effect of $\operatorname{EcR}^{\mathrm{DN}}$ on neuronal morphology. a, Morphology of lamina

722 neurons (L1-L5) in wildtype (WT) brains and upon pan-lamina expression of EcR ${ }^{\mathrm{DN}}, \mathrm{EcR}^{\mathrm{DN}}+$

723 EcR-B1 cDNA and EcR RNAi. mAb24B10 labels M3 and M6. M3 is highlighted with yellow

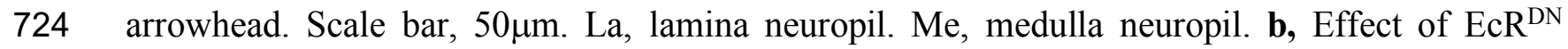

725 expression on morphology of T4/T5 neurons. Four layers in the lobula plate, a, b, c and d, are

726 marked with red, yellow, blue and pink arrowheads, respectively. Cartoons of one T4 (purple

727 neuron, top) and one T5 neuron (purple neuron, bottom) are shown to highlight wildtype

728 morphology. Scale bar, $20 \mu \mathrm{m}$. 
a

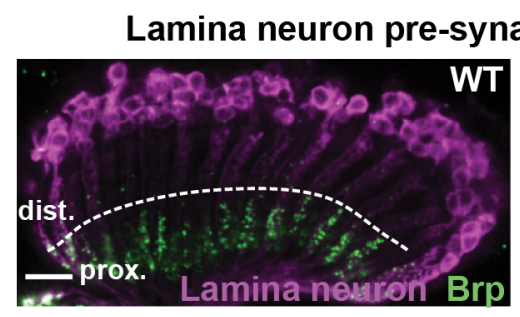

b

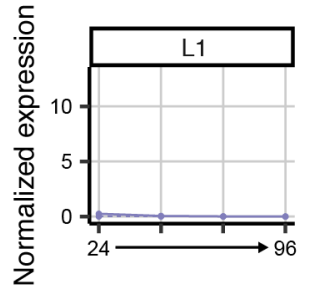

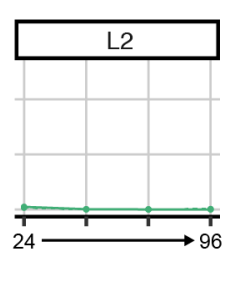
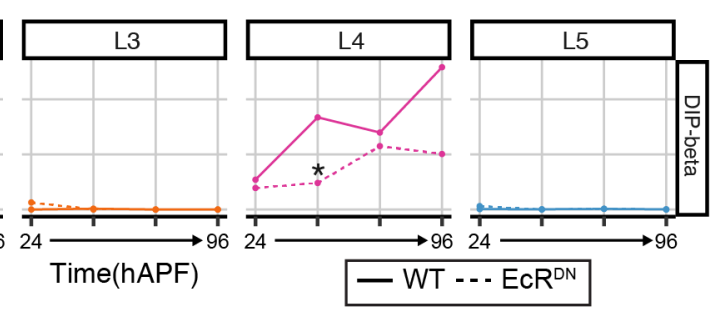

Extended data Fig. 15 | Distribution of presynaptic sites in the lamina $\pm \mathbf{E c R}^{\mathrm{DN}}$ expression.

730 a, Distribution of Brp puncta (presynaptic sites) in the lamina neuropil for wildtype (WT) and

731 EcR $^{\mathrm{DN}}$-expressing lamina neurons. Magenta, all lamina neurons, Green, Brp puncta. Dotted line

732 differentiates proximal and distal halves of the lamina neuropil (see Methods). Scale bars, $50 \mu \mathrm{m}$.

733 Distribution of distal BRP puncta/ cartridge for both conditions is also shown. ***, student's t test

734 p-value $<0.001$. n, number of cartridges quantified ( 7 animals for wildtype, 6 animals for $\mathrm{EcR}^{\mathrm{DN}}$ ).

735 b, Normalized expression of DIP- $\beta$ in wildtype (solid lines) and EcR ${ }^{\text {DN }}$-expressing L1-L5 neurons

736 (dashed lines). ${ }^{*}$, p-value $\leq 0.05$, fold change $\geq 2$. 
a
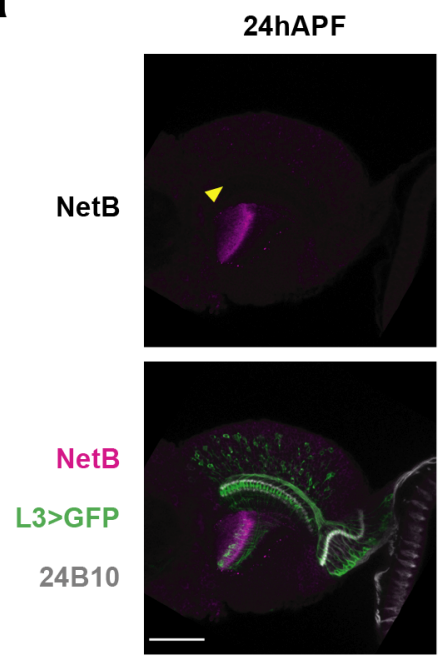

b

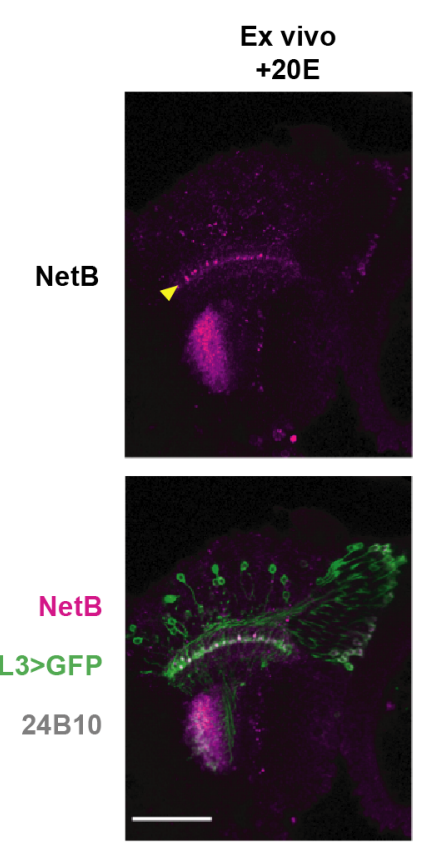

48hAPF
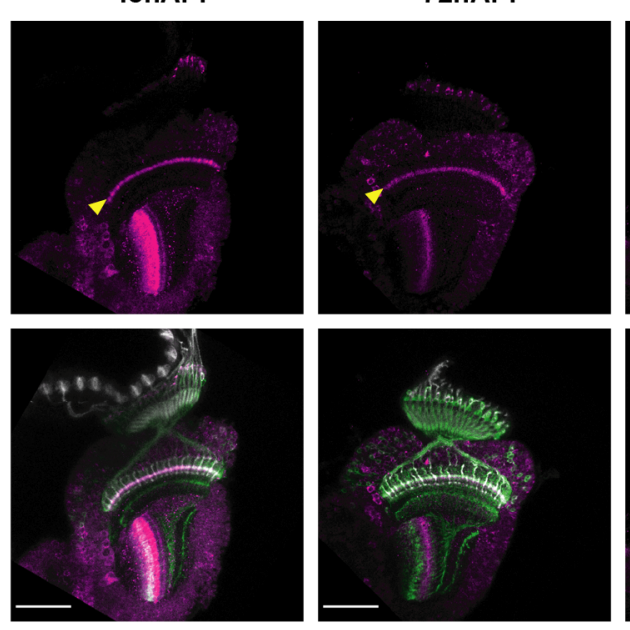

Adult
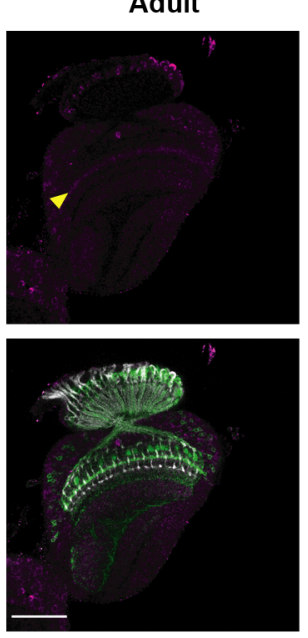

C P-value of KS test for R8 phenotype distribution
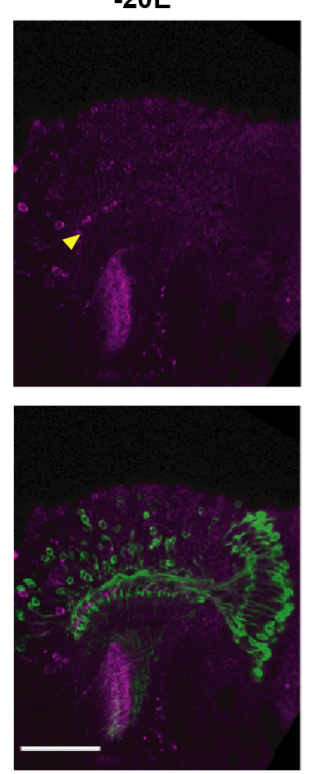

\begin{tabular}{|c|c|c|c|c|}
\hline & WT & $\begin{array}{c}\text { L3> } \\
\text { EcR }^{\mathrm{DN}}\end{array}$ & $\begin{array}{l}\text { L3> } \\
\text { NetB }\end{array}$ & $\begin{array}{c}\text { L3> } \\
\text { EcR } \\
\text { NetB }\end{array}$ \\
\hline WT & 1 & $<10^{-15}$ & 0.65 & $2.2 \times 10^{-15}$ \\
\hline $\begin{array}{l}\text { L3> } \\
\text { EcR }^{\text {DN }}\end{array}$ & $<10^{-15}$ & 1 & $<10^{-15}$ & $<10^{-15}$ \\
\hline $\begin{array}{l}\text { L3> } \\
\text { NetB }\end{array}$ & 0.65 & $<10^{-15}$ & 1 & $<10^{-15}$ \\
\hline $\begin{array}{c}\text { L3> } \\
\text { EcR } \\
\text { NetB } \\
\text { NetB }\end{array}$ & $2.2 \times 10^{-15}$ & $<10^{-15}$ & $<10^{-15}$ & 1 \\
\hline
\end{tabular}

\section{Extended data Fig. 16 | NetB expression dynamics and dependence on Ecdysone for} Adult. M3 is highlighted with a yellow arrowhead. mAb24B10 is used as a layer marker in the

740 medulla. Note that maximum expression in M3 is seen at 48hAPF. b, Anti-NetB antibody staining

741 (magenta) in ex vivo cultured brains \pm inclusion of $20 \mathrm{E}$ in the medium (see Methods and Extended

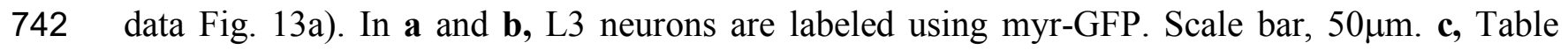


bioRxiv preprint doi: https://doi.org/10.1101/2020.09.18.304410; this version posted September 19, 2020. The copyright holder for this preprint (which was not certified by peer review) is the author/funder, who has granted bioRxiv a license to display the preprint in perpetuity. It is made available under aCC-BY-NC-ND 4.0 International license.

743 showing p-values from KS test for comparison of R8 axon depth distributions between all genetic

744 conditions in Fig. 3c. Note that $\mathrm{p}$-value $=2.2 \mathrm{X} 10^{-15}$ for comparison between $\mathrm{L} 3>\mathrm{EcR}^{\mathrm{DN}}+\mathrm{NetB}$

745 and WT is indicative of an incomplete rescue of $\mathrm{EcR}^{\mathrm{DN}}$ phenotype by NetB overexpression. 

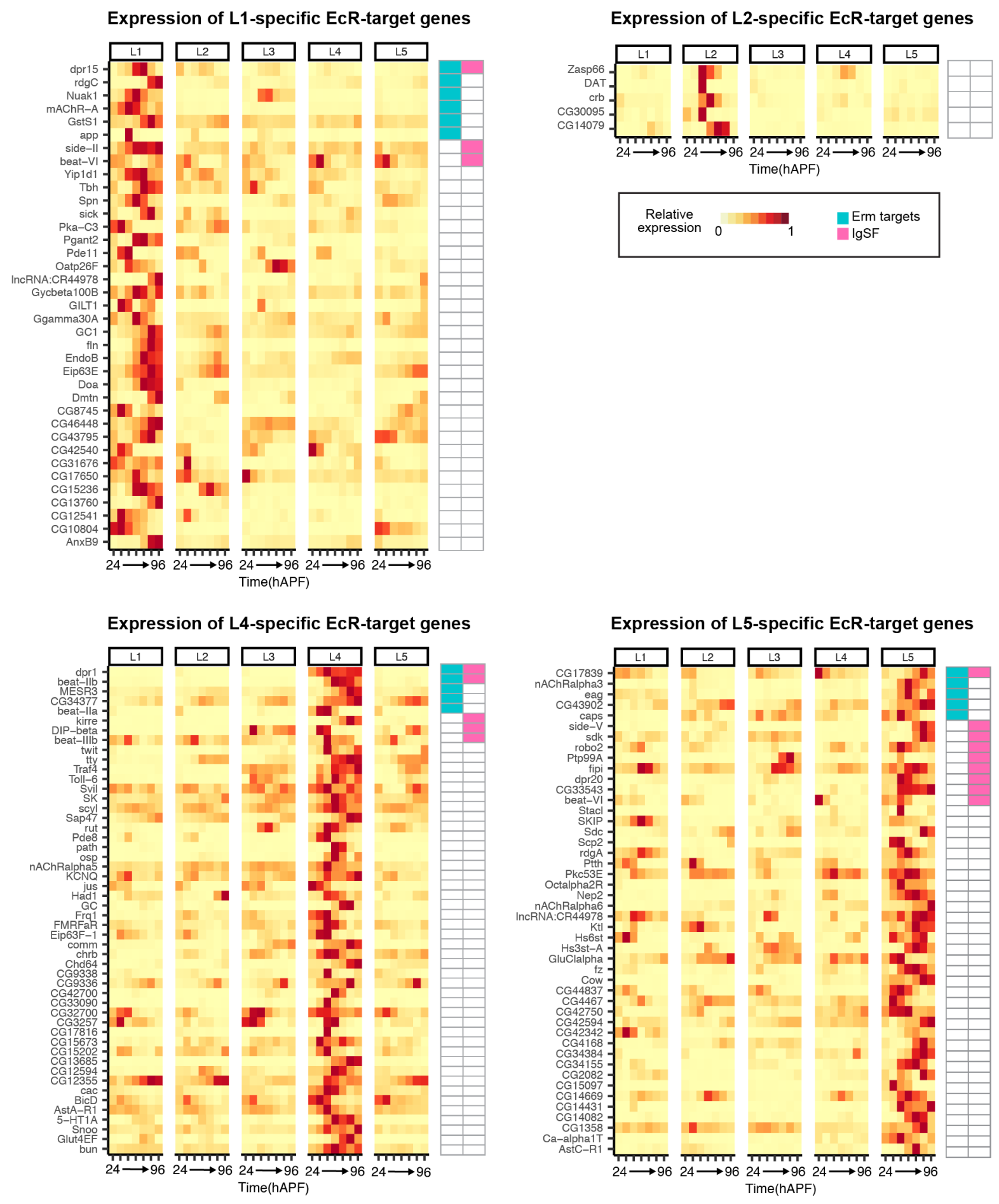

746 Extended data Fig. $17 \mid$ L1, L2, L4 and L5 - specific targets of EcR. Heat map showing relative

747 expression of L1, L2, L4 and L5-specific targets of EcR (see Methods). Aqua, targets of

748 transcription factor Erm identified in Peng et al. ${ }^{32}$. Magenta, Immunoglobulin Superfamily genes. 
bioRxiv preprint doi: https://doi.org/10.1101/2020.09.18.304410; this version posted September 19,2020 . The copyright holder for this preprint (which was not certified by peer review) is the author/funder, who has granted bioRxiv a license to display the preprint in perpetuity. It is made available under aCC-BY-NC-ND 4.0 International license.

749 The relatively small number of L2-specific targets of EcR is likely to reflect low expression of

$750 \quad \mathrm{EcR}^{\mathrm{DN}}$ in these neurons (Extended data Fig. 7e). 


\section{Supplementary Tables}

752 Supplementary Table 1. Fly strains used in this study

753 Supplementary Table 2. Developmental transcriptome of lamina neurons. Normalized

754 expression values are given for all expressed genes.

755 Supplementary Table 3. Transcriptome of L1 neurons at 40hAPF, 60hAPF and 72hAPF by bulk

756 RNA-Seq. Numbers in the table are RPKM values.

757 Supplementary Table 4. Gene groups generated by k-means clustering. Relative expression

758 values are given for all expressed genes.

759 Supplementary Table 5. Gene Ontology (GO) analysis of gene groups generated by k-means

760 clustering.

761 Supplementary Table 6. TF binding site analysis using i-cisTarget (see Methods).

762 Supplementary Table 7. ATAC-Seq analysis of L1 neurons at 40hAPF, 60hAPF and 72hAPF.

763 Measure of reads mapped to each peak are given as RPKM values. Peaks with differential 764 accessibility between consecutive time points are also given.

765 Supplementary Table 8. Normalized expression in $\mathrm{WT}$ and $\mathrm{EcR}^{\mathrm{DN}}$-expressing lamina neurons.

766 Genes differentially expressed between $\mathrm{WT}$ and $\mathrm{EcR}^{\mathrm{DN}}$ are also given.

767 Supplementary Table 9. Gene Ontology (GO) analysis of common and cell-type dependent 768 targets of EcR.

769 Supplementary Table 10. Normalized expression in wRNAi and EcR RNAi-expressing lamina

770 neurons. Genes differentially expressed between wRNAi and EcR RNAi are also given.

771 Supplementary Table 11. Normalized expression in wRNAi and Hr3 RNAi-expressing lamina

772 neurons. Genes differentially expressed between wRNAi and Hr3 RNAi are also given. 
bioRxiv preprint doi: https://doi.org/10.1101/2020.09.18.304410; this version posted September 19, 2020. The copyright holder for this preprint (which was not certified by peer review) is the author/funder, who has granted bioRxiv a license to display the preprint in perpetuity. It is made available under aCC-BY-NC-ND 4.0 International license.

773 Supplementary Table 12. Lists of genes likely to be involved in step 1 or step 2 of L5 axonal

774 morphogenesis (see Fig. 4c). 


\section{Acknowledgements}

776 We thank Dr. Pecot (Harvard), Dr. Yamanaka (UC Riverside), Dr. Black (UCLA), Dr. De Robertis

777 (UCLA), Dr. Riddiford (Univ. of Washington) and Dr. Truman (Janelia Research Campus) for

778 helpful discussions. We thank Dr. Schuldiner (Weizmann Institute of Science) and members of the

779 Zipursky lab for feedback on the manuscript, and Dr. Diaz de la Loza for help with figure

780 illustrations. We would like to specifically acknowledge Juyoun Yoo’s (Zipursky Lab) and Rachel

781 Hodge's (Jones Lab, UCLA) help with ATAC-Seq library prep and immunostaining. We also

782 thank the BSCRC Sequencing Core (UCLA) and the TCGB core (UCLA) for help with library 783 preparation and sequencing; the BSCRC FACS core (UCLA) and the Witte lab (UCLA) for

784 assistance with FACS purification of lamina neurons; and the IDRE Statistics Consulting (UCLA)

785 and Dr. Balliu (UCLA) for assistance with the statistical analysis of data. Reagents provided by

786 Dr. Akin (UCLA), Dr. Laski (UCLA), Dr. Wang (Duke-NUS), Dr. Pecot (Harvard), Dr. Thummel

787 (Univ. of Utah) and Dr. Bashaw (Univ. of Penn.) were critical for this work. This work was

788 supported by NIH T32-NS048004 Neurobehavioral Genetics Training Grant (S.J.), Helen Hay

789 Whitney Foundation (S.J.) and Whitcome Fellowship (Y.L.). S.L.Z is an investigator of the 790 Howard Hughes Medical Institute.

\section{Author contributions}

792 S.J., Y.L., Y.K. and S.L.Z. designed experiments. S.J., Y.L., P.M. and B.P. acquired data. S.J.,

793 Y.L. and Y.K. analyzed the data. S.J., Y.L. and S.L.Z. wrote the manuscript with input from all 794 co-authors. 
bioRxiv preprint doi: https://doi.org/10.1101/2020.09.18.304410; this version posted September 19, 2020. The copyright holder for this preprint

(which was not certified by peer review) is the author/funder, who has granted bioRxiv a license to display the preprint in perpetuity. It is made available under aCC-BY-NC-ND 4.0 International license.

\section{Competing interest declaration}

796 The authors declare no competing interests.

\section{Additional information}

798 Supplementary Information is available for this paper.

799 Correspondence and requests for materials should be addressed to: Dr. S. Lawrence Zipursky -

800 lzipursky@mednet.ucla.edu. 


\section{$801 \quad$ Additional References}

802 50. Hoskins, R. A. et al. The Release 6 reference sequence of the Drosophila melanogaster

803 genome. Genome Res 25, 445-458 (2015).

804 51. Picelli, S. et al. Full-length RNA-seq from single cells using Smart-seq2. Nat Protoc 9, 171-

$805181(2014)$.

806 52. Buenrostro, J. D., Giresi, P. G., Zaba, L. C., Chang, H. Y. \& Greenleaf, W. J. Transposition

807 of native chromatin for fast and sensitive epigenomic profiling of open chromatin, DNA-binding

808 proteins and nucleosome position. Nat Methods 10, 1213--1218 (2013).

809 53. Heinz, S. et al. Simple Combinations of Lineage-Determining Transcription Factors Prime

810 cis-Regulatory Elements Required for Macrophage and B Cell Identities. Mol Cell 38, 576-589

811 (2010).

812 54. Xu, S. et al. Interactions between the Ig-Superfamily Proteins DIP- $\alpha$ and Dpr6/10 Regulate

813 Assembly of Neural Circuits. Neuron 100, 1369--1384.e6 (2018). 